\title{
An analytical model for accurate and numerically efficient tooth contact analysis under load, applied to face-milled spiral bevel gears
}

\author{
M. Vivet ${ }^{\mathrm{a}, \mathrm{b}, \mathrm{c}, *}$, D. Mundo ${ }^{\mathrm{a}}$, T. Tamarozzi ${ }^{\mathrm{b}, \mathrm{c}}, \mathrm{W}$. Desmet ${ }^{\mathrm{c}, \mathrm{d}}$ \\ ${ }^{a}$ Department of Mechanical, Energy and Management Engineering, University of Calabria, Ponte P. Bucci Cubo 46C, 87036 \\ Arcavacata di Rende (CS), Italy \\ ${ }^{b}$ Siemens Industry Software NV, Interleuvenlaan 68, B-3001 Leuven, Belgium \\ ${ }^{c}$ Department of Mechanical Engineering, KU Leuven, Celestijnenlaan 300B, B-3001 Leuven, Belgium \\ ${ }^{d}$ DMMS Core Lab, Flanders Make, Belgium
}

\begin{abstract}
An analytical model that allows for a computationally efficient analysis of face-milled spiral bevel gears, is presented. The methodology builds on the consideration that the mating tooth flanks are designed to transmit motion in a nearly conjugate manner. A multibody approach to tooth contact analysis is proposed that assumes contact between rigid surfaces. By taking advantage of the action surfaces for each flank pair, contact is detected in a computationally efficient and accurate way. An analytical load distribution model is used to translate the detected penetration into resulting contact forces, under the assumption that the flank penetration matches the deformation of the teeth if they were flexible. To account for the global tooth deformation Tredgold's approximation in combination with a set of expressions based on beam theory are utilized, while the local contact deformation is modeled based on Hertz theory. The methodology is validated against nonlinear finite element simulations. A comparison in terms of transmission error, contact patterns and contact pressure is provided. Contrary to FE simulations the proposed methodology requires significantly less computational effort, allowing further extension to optimization or system analysis problems.
\end{abstract}

Keywords: tooth contact analysis, equation of meshing, bevel gears, static transmission error 2010 MSC: 68U07, 68U20, 74B20, 74S05

\section{Introduction}

Increasingly stringent regulations related to energy efficiency and emissions have made transmission design ever more challenging, as the industry is forced to find answers to conflicting design requirements such as increased performance in terms of power density and durability, lower noise emissions and higher 5 mechanical efficiency. The optimization process that is required to improve any of the above design criteria for spiral bevel gear pairs, is hindered by the complexity of contact phenomena at play during operation. Moreover, while the tooth geometry of an individual gear is determined by its manufacturing process, the resulting contact characteristics are always the result of interactions between both gear pair elements. Because of this a lot of research efforts have already gone into the analysis and optimization of individual gear pairs either by experimental testing or by computerized tooth contact analysis (TCA) [1] - [16].

Through simulation of the gear meshing process TCA aims at providing answers about the key performance characteristics of the gear pair without the need for expensive prototyping and experimental testing. The performance of a spiral bevel gear pair is typically classified based on the quality of the transmission

\footnotetext{
* Corresponding author

Email address: mathijs.vivet@kuleuven.be (M. Vivet)
} 
error (TE), the contact point path (CPP) on the gear flanks, the contact zone under load and the sensitiv15 ity of these characteristics w.r.t. configurational misalignments [17]. Common practice has been to extend the TCA with finite element analysis (FEA) of the gear pair in order to assess its quality under loaded conditions. Yet, time-consuming model creation and heavy computational requirements make them impractical for parameter studies or optimization campaigns. Furthermore, when it comes to the simulation of a transmission as a whole, numerical methods should not only be able to simulate the behavior of individual gear pairs but also their interactions with other drivetrain components. Starting from these considerations, the presented work focuses on the development of an accurate and computationally efficient methodology for the three dimensional contact simulation of spiral bevel gears. The result of these efforts is a toolchain of methods that allows: geometry creation, finite element model generation, TCA in both unloaded and loaded conditions and that opens up possibilities for further usage in optimization strategies or dynamic 25 simulations of drivetrain systems.

\subsection{Methodologies for unloaded tooth contact analysis}

The groundwork for most of today's methods for geometry, manufacturing and tooth contact analysis for spiral bevel and hypoid gears, has been laid by Wildhaber [18] and Baxter [19]. In the following years significant advancements were made by the researchers of the Gleason Works [20] and Klingelnberg-Oerlikon [21]. Their efforts resulted in the creation of commercial TCA solutions for spiral bevel and hypoid gears. Litvin, Fuentes and Argryis used a method of local synthesis to optimize the machines settings in order to obtain predetermined contact characteristics for the gear pair at a predefined point [1] - [3]. The meshing of the gear flanks under no-load is based on the condition of continuous tangency between the mating flanks. This condition mathematically translates to identifying contact points as those points on the corresponding 35 flanks that become coincident and whose surface normal vectors become collinear in case of contact [17]. They were able to determine the unloaded transmission error (UTE), the path of contact points and the instantaneous contact lines. The length and direction of the contact were obtained through the use of contact ellipses, centered around the contact point, for which the size was computed based on the principal curvatures and directions of the contacting surfaces. The condition of continuous tangency results in a system of nonlinear equations that has to be solved in order to identify the contact points. Finding an accurate solution to this problem is complicated as convergence to points in the proximity of the true contact point can occur due to the high-level of conformity (similarity) between the mating flanks [22]. Strategies for automatic determination of appropriate start values have been proposed by Litvin et al. for normal TCA [23] and more recently by He et al. for TCA in the presence of misalignment errors [24]. Simon 45 developed a methodology for TCA, which he applied to the analysis of spiral bevel gears [6, 7] and hypoid gears with optimized contact pressure and transmission error $[4,5,8]$. The TCA problem was also defined through the coincidence of contact points and collinearity of surface normal vectors. By assuming that under load the contact point would evolve into a line contact, he was able to compute the instantaneous contact lines through the minimization of the separation function, while avoiding the computation of the relative curvatures [6, 7]. Stadtfeld introduced the concept of ease-off topography to TCA in [25] and used it in a later study to optimize the shape of the UTE by applying tooth flank modifications to reduce the effects of tooth impacts and lower the noise of the gear pair [9]. His definition for ease-off topography includes the effects of flank form corrections and misalignments, applied to either pinion or gear, that cause a mismatch between the mating flanks and therefore a non-conjugate behavior of the gear pair. Kolivand and Kahraman 55 developed a novel methodology for TCA which was based on the ease-off concept. During the unloaded tooth contact analysis (UTCA) they constructed the surface of roll angles and ease-off topography from the gear pair installment, whether or not misaligned. Through the combination of the surface of roll angles and the ease-off topography they were able to compute potential contact curves and unloaded transmission error in an elegant way $[10,22]$. In their developed approach the ease-off topography was defined w.r.t. the gear

60 element. A similar method for ease-off based TCA was used by Fan to optimize the pinion tip line to avoid root-tip interference [12] and by Dooner et. al for the analysis of spiral bevel gears with kinematically correct motion transfer [26]. Artoni et al. developed a TCA variant that expressed the ease-off topography w.r.t. the pinion element, allowing corrective modifications to the behavior of the gear pair by only correcting the pinion [16]. 


\subsection{Methodolgies for loaded tooth contact analysis}

Loaded tooth contact analysis (LTCA) models for spiral bevel and hypoid gears are relatively scarce. Krenzer proposed a methodology for LTCA in which the overall tooth deformation was modeled as a combination of Hertzian contact theory and beam theory. In his work a TCA program, based on continuous tangency, was used to describe the tooth geometry and no-load contact analysis [27]. Elkholy et al. used an

70 analytical procedure to calculate the transmitted load distribution for straight bevel gears by dividing the teeth into different slices with varying cross-section [28]. Using Tredgold's approximation he assumed that the cross-section of each slice could be well represented by involute tooth profile, for which he computed the deflection using analytical formulas developed by Nakada and Utagawa [29]. Gosselin et al. developed a LTCA program for spiral bevel and hypoid gears and included tooth compliance by precomputing the tooth 75 stiffness along the surface normal with a three dimensional finite element (FE) model of a single tooth. Weighting functions were used to translate the tooth compliance values, computed on the FE nodes, to the contact points [30]. Fan and Wilcox presented a methodology for loaded TCA where the loaded contact is assumed to take place along the conjugate line of tooth contact. The concept of a combined flexibility matrix is used to model the compliance of the gear, the pinion and the housing. Load sharing is included through

so the use of FEA meshing models with multiple teeth [31]. Hemmelmann also developed a FE-based LTCA model for spiral bevel and hypoid gears where he computed tooth compliance from a three-dimensional FE model that included multiple teeth [32]. He used the spring-model by Neupert [33], to include the coupling effects between teeth. Kolivand and Kahraman used a semi-analytical approach, developed by Vaidyanathan et al.[34], to model the tooth stiffness by using a Rayleigh-Ritz based shell model with linearly varying tooth thickness along the toe, heel and top [10,22].

\subsection{Scope and structure}

A numerically efficient methodology is proposed to determine contact between multiple meshing flanks under loaded conditions. The equation of meshing, which originates from differential geometry, together with the ideas for TCA, developed by Kolivand in [10] and [22], will prove essential in the creation of an efficient penetration-based contact detection algorithm. The methodology is formulated in such a way that it can be easily augmented to include gear pair misalignment or extended towards the simulation of spiral bevel gear pair dynamics within a multibody framework. The current work focuses on the development and validation of the methodology for the loaded nominal configuration, keeping gear pair misalignments and dynamical aspects for future work. Global tooth deformation is included through a set of analytical expressions of [29] and [28] that are applied for the first time to the approximation of the tooth stiffness of spiral bevel gear teeth. Additionally, all the presented methods have been combined in a dedicated toolchain for the analysis of spiral bevel gear pairs that covers: (i) gear flank geometry computation, (ii) automated FE model creation, (iii) automated pre-processing of the required data for contact detection, (iv) UTCA and (v) LTCA. The presented methodology is applied and validated with the analysis of a spiral bevel gear pair that has also been described in literature [2].

An accurate contact detection between two meshing geometries requires a correct description of the respective geometry. A concise overview of the generation process for the tooth surface geometry of face-milled spiral bevel gears is therefore presented in Section 2. A general method to compute the principal curvatures of the tooth is proposed. Section 3 briefly explains the theory of conjugate surfaces and the creation of the surface of roll angles and the action surface. In Section 4 the information that was predetermined during the unloaded tooth contact analysis is used to create an efficient contact detection algorithm that detects contact over multiple tooth pairs. The tooth deflections under load are computed by using a penetration-based contact model that applies a slicing technique to compute the individual tooth slice deformation based on Hertzian contact and beam theory. Finally, in Section 5 the results for loaded transmission error, overall contact pattern and maximum contact pressure are presented. The results of the proposed model are then validated against results that were obtained with NX Nastran (Solution 601), a commercial software package for nonlinear finite element analysis. 


\section{Geometry of face-milled spiral bevel gears}

On an industrial scale, spiral bevel gears are typically manufactured by either a face-milling (FM) or a face-hobbing $(\mathrm{FH})$ process. Face milling is a single indexing process during which the blade groups cut one tooth at the time. Once the tooth is completed, the cutter head withdraws and the gear blank indexes with one pitch. This process is repeated until all the teeth of the gear are cut. Face-hobbing on the other hand is a continuous indexing process where the blade groups on the cutter head cut different parts of different teeth at the same time. In this work a face-milled spiral bevel gear pair is analyzed for which the geometry was obtained by simulating the final steps of the manufacturing process.

\subsection{Gear flank geometry}

The manufacturing of a real spiral bevel gear pair consists of five independent steps (five-cut process) [25]: (i) gear roughing, (ii) gear finishing, (iii) pinion roughing, (iv) pinion finishing by using only the inner blades (convex) and (v) pinion finishing by using only the outer blades (concave). Computerized models, which are used to generate the tooth flank surfaces simulate only the finishing steps of the manufacturing process. The model that has been used here is based on the older cradle-based hypoid generators of which the underlying mathematical model has already been extensively described in literature. The reader is referred to the works $[2,3,17]$ for a more detailed description.

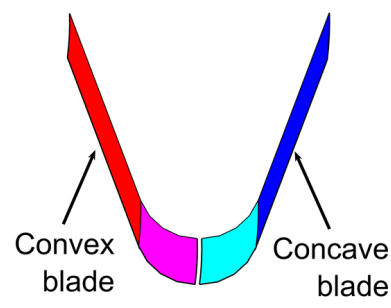

(a) Cutter blade edges

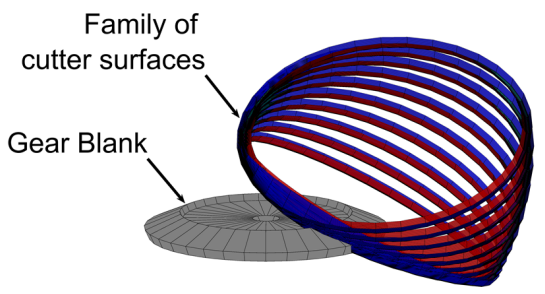

(b) Cutting process

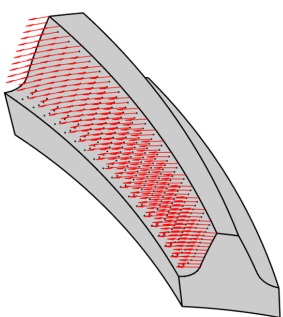

(c) Gear tooth surface

Figure 1: Simulation of the face-milling process.

A virtual model of both gear and pinion is created, following the steps of Figure 1. For this gear pair a cutter blade with a straight blade edge and circular fillet has been used (Figure 1a). By tracking the motion of the blade surfaces a family of cutter surfaces is created as they pass through the gear blank (Figure 1b). In case of a cradle-based hypoid generator the family of cutter surfaces is described by the rotation of the cradle angle, the rotation of the cutter head and the location on the blade. The resulting tooth geometry is defined by the envelope to this family of surfaces and is obtained as the solution to the equation of meshing between the moving cutter surface and the gear blank, together with two additional constraints that define the location on the tooth flank [17]. The gear tooth surfaces are computed as an ordered set of points and their corresponding surface normal vectors (Figure 1c).

\subsection{Principal curvatures and directions}

Knowing the principal curvatures and corresponding directions allows to reconstruct the curvature along any given direction $\boldsymbol{t}$. The principal curvatures at a point on the surface are found as the extreme values of the curvature at this point. To compute the principal curvature and directions at a point $P$ that lies on the surface $\Sigma$, this work uses the methodology of [35]. In the vicinity of a point $P$ the curvature along a given direction $\boldsymbol{t}$ can be well approximated by fitting a spatial circle through $P$. Considering that the surface $\Sigma$ was discretized as an ordered set of surface points and surface normal vectors, a 3 -by-3 grid is defined with $P$ as central point. This allows to construct four circles $c_{i}(i=1,4)$ along different directions through $P$, as shown in Figure 2. Each circle $c_{i}$ is defined by a center point $C_{i}$ and a radius $R_{i}$ and runs through $P$ where a local reference frame is defined. The unit vectors $\boldsymbol{n}_{i}, \boldsymbol{t}_{i}$ and $\boldsymbol{b}_{i}$ are the curve normal vector, the curve tangent vector and the curve binormal vector of the circle $c_{i}$, respectively. The curvature 


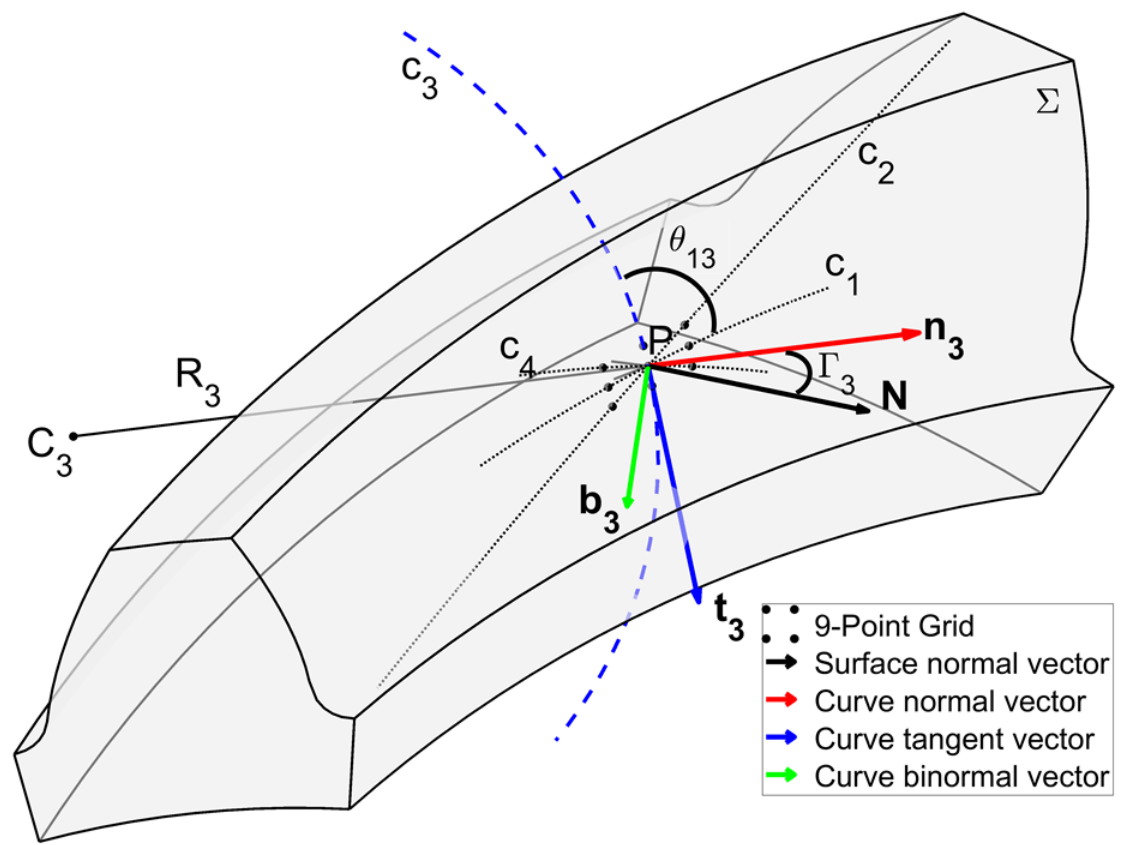

Figure 2: Surface normal curvature calculation at the Point $P$ by fitting circles $c_{i}$ along different directions. The process is illustrated for circle $c_{3}(i=3)$.

of the spatial curve is computed from the radius of curvature $r_{c, i}$ as $\kappa_{c, i}=1 / r_{c, i}$ with $r_{c, i}=R_{i}$. The surface normal curvature $\kappa_{n, i}$ is obtained for each circle by projecting the curvature of the curve $\kappa_{c, i}$ onto the surface normal vector $\boldsymbol{N}$, with $\Gamma_{i}$ being the projection angle between $\boldsymbol{n}_{i}$ and $\boldsymbol{N}$.

$$
\kappa_{n, i}=\kappa_{c, i} \boldsymbol{n}_{i} \cdot \boldsymbol{N}=\kappa_{c, i} \cos \left(\Gamma_{i}\right)
$$

Once at least three normal curvatures along three different directions at $P$ are known the Mohr circle can be used to compute the principal curvatures and directions [35, 36]. The Mohr circle of curvature, given by Equations (2) and (3), expresses the relationship between the surface normal curvature $\kappa_{n, i}$, the surface torsion $\tau_{g, i}$ and the minimum and maximum principal curvatures $\kappa_{\min }$ and $\kappa_{\max }$. This relationship is found as a function of $\theta_{i}$, which represents the angle between the direction of $\kappa_{\max }$ and the direction of the curve $c_{i}$.

$$
\left\{\begin{aligned}
\kappa_{n, i} & =\frac{1}{2}\left(\kappa_{\max }+\kappa_{\min }\right)+\frac{1}{2}\left(\kappa_{\max }-\kappa_{\min }\right) \cos \left(2 \theta_{i}\right) \\
\tau_{g, i} & =-\frac{1}{2}\left(\kappa_{\max }-\kappa_{\min }\right) \sin \left(2 \theta_{i}\right)
\end{aligned}\right.
$$

The circle of Mohr is reconstructed by solving a system of three nonlinear equations, obtained by using Equations (1) and (2) along three different directions, while also accounting for the angle $\theta_{1 i}$ between the direction of $c_{i}$ and a chosen direction of $c_{1}$. Figure 2 provides an example for the angle $\theta_{1 i}$ for $i=3$. Euler's formula, given by Equations (4) and (5), can then be used to compute the surface normal curvature $\kappa_{n}$ along any direction at the point $P$, with $\theta_{t}$ the angle between the direction of curvature $\boldsymbol{t}$ and the direction of maximum principal curvature $\boldsymbol{t}_{\max }$.

$$
\left\{\begin{aligned}
\kappa_{n} & =\kappa_{\max } \cos ^{2}\left(\theta_{t}\right)+\kappa_{\min }\left(1-\cos ^{2}\left(\theta_{t}\right)\right) \\
\cos \left(\theta_{t}\right) & =\boldsymbol{t} \cdot \boldsymbol{t}_{\max }
\end{aligned}\right.
$$




\section{Computation of the conjugate surfaces}

The numerical efficiency of the contact detection strategy that is developed in this work, is achieved thanks to the use of precomputed information about the gear pair. More specifically, the surface of roll angles, surface of action and the conjugate flanks to the real mating gear flanks are computed prior to the contact simulation. To this end, the UTCA methodology of [22] is modified and extended to construct the conjugate surfaces and gear-based ease-off topography from predefined tooth flanks of the pinion and gear elements without the need for additional knowledge about the manufacturing process. The usefulness of this extension has been demonstrated in [26]. A description of the most important steps of the methodology is given in this section before moving to contact detection under load.

\subsection{Equation of meshing}

Similarly to how the cutter blade meshes perfectly with the gear blank while it performs the cutting of the tooth flank, a real tooth flank also meshes perfectly with its conjugate when it rotates around its rotational axis. The conjugate to a given flank is therefore defined as the envelope to a family of real tooth flank surfaces and computed using the equation of meshing for the real flank while taking into account the relative position and orientation of the gear pair. The equation of meshing, which originates from Differential Geometry [17], is for practical reasons often reformulated as:

$$
\boldsymbol{N}\left(\phi_{z}\right) \cdot \boldsymbol{v}\left(\phi_{z}\right)=0
$$

For any point $P$ on a given surface $\Sigma$, the condition of perfect meshing, given by Equation (6), is thus obtained when there is neither penetration nor gap between the point $P$ and a coincident point on the mating surface, conjugate to $\Sigma$. In such a case the relative velocity $\boldsymbol{v}$ between the point $P$ and the coincident point on the mating surface, will be perpendicular to the surface normal vector $\boldsymbol{N}$ at the point $P$.

The presented methodology makes two assumptions regarding the gear geometry when solving the equation of meshing: (i) all teeth on both the pinion and the gear are identical without indexing errors and (ii) the gear geometry is predefined. The first assumption allows to solve the equation of meshing for the flanks of a single tooth pair, the reference tooth pair, and to translate the results to other tooth pairs when needed. Considering the gear flank geometry as predefined means that solving Equation (6) is reduced to nothing more than finding the solution in terms of rotational angle $\phi_{z}$ to a single nonlinear equation at each point of the gear flank surface.

\subsubsection{Position and orientation of the gear pair}

Figure 3 shows the different reference frames that describe the position and orientation of the gear pair. The surface points of the pinion flank (gear 1 ) are expressed in a local reference frame $S_{1}$, while the points of both the conjugate to the pinion flank and the gear flank (gear 2$)$ are expressed in the local reference frame $S_{2}$. The gear pair installment is defined through the shaft offset $E$, the pinion axial offset $A_{1}$, the gear axial offset $A_{2}$ and shaft angle $\gamma$. The angles $\phi_{z 1}$ and $\phi_{z 2}$ represent the rotation about the rotational axes of the pinion and of the gear w.r.t. the base frame, respectively. The base frame $S_{B}$ is introduced as an auxiliary reference frame to describe the relative position and orientation of the gear and the pinion. It is defined in such a way that its origin stays the same as that of $S_{1}$ while the pinion is only allowed to rotate about their common z-axis. The homogeneous transformation matrix $\mathbf{T}_{B 1}$ expresses the position and orientation of the reference frame $S_{1}$ w.r.t. the base frame $S_{B}$, while matrix $\mathbf{T}_{B 2}$ expresses the position and orientation of the reference frame $S_{2}$ w.r.t. the base frame $S_{B}$. The two transformation matrices are given by Equation (7) and Equation (8), respectively.

$$
\begin{aligned}
& \mathbf{T}_{B 1}=\mathbf{T}_{z}\left(\phi_{z 1}\right) \\
& \mathbf{T}_{B 2}=\mathbf{T}_{B 2^{\prime}}\left(A_{1}, \gamma, E, A_{2}\right) \mathbf{T}_{z}\left(\phi_{z 2}\right)
\end{aligned}
$$




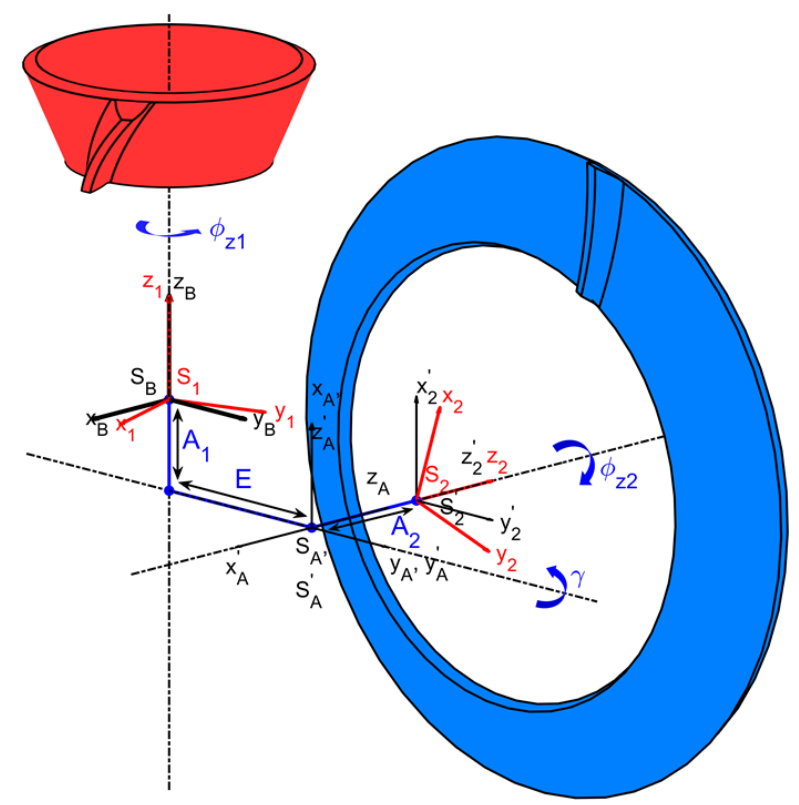

Figure 3: Relative orientation of Gear1 (pinion) and Gear2 (gear) w.r.t. the base frame, displayed for installment parameters $A_{1}>0, E>0, A_{2}>0$ and $\gamma=-90 \mathrm{deg}$.

The transformation matrix $\mathbf{T}_{z}$ represents the rotation about the gear rotational axis for either the pinion in Equation (7) or for the gear in Equation (8).

$$
\mathbf{T}_{z}\left(\phi_{z}\right)=\left[\begin{array}{cccc}
\cos \left(\phi_{z}\right) & -\sin \left(\phi_{z}\right) & 0 & 0 \\
\sin \left(\phi_{z}\right) & \cos \left(\phi_{z}\right) & 0 & 0 \\
0 & 0 & 1 & 0 \\
0 & 0 & 0 & 1
\end{array}\right]
$$

The considered gear pair installment parameters are combined in a single transformation matrix $\mathbf{T}_{B 2^{\prime}}$, given by Equation (10).

$$
\mathbf{T}_{B 2^{\prime}}=\left[\begin{array}{cccc}
\cos (\gamma) & 0 & \sin (\gamma) & 0 \\
0 & 1 & 0 & 0 \\
-\sin (\gamma) & 0 & \cos (\gamma) & -A_{1} \\
0 & 0 & 0 & 1
\end{array}\right]\left[\begin{array}{cccc}
1 & 0 & 0 & 0 \\
0 & 1 & 0 & E \\
0 & 0 & 1 & A_{2} \\
0 & 0 & 0 & 1
\end{array}\right]
$$

\subsubsection{The conjugate to a real tooth flank}

The conjugate to a real tooth flank is obtained by applying the equation of meshing, while accounting for the relative position and orientation of the gear pair. Taking the pinion as an example, the equation of meshing between the real pinion flank and its theoretical conjugate, given in Equation (11), is obtained by expressing Equation (6) w.r.t. the base frame $S_{B}$.

$$
\boldsymbol{N}_{B}^{(1)} \cdot\left[\left(\boldsymbol{\omega}_{B}^{(1)} \times \boldsymbol{r}_{B}^{(1)}\right)-\left(\boldsymbol{\omega}_{B}^{(2)} \times \boldsymbol{\rho}_{B}\right)\right]=0
$$

The relative velocity $\boldsymbol{v}_{B}^{(12)}=\left(\boldsymbol{\omega}_{B}^{(1)} \times \boldsymbol{r}_{B}^{(1)}\right)-\left(\boldsymbol{\omega}_{B}^{(2)} \times \boldsymbol{\rho}_{B}\right)$ defines the correct kinematic relation between the surface points of the pinion and its conjugate flank. The vector $\boldsymbol{\rho}_{B}=\boldsymbol{r}_{B}^{(1)}-\boldsymbol{S}_{B}^{(B 2)}$ accounts for the rotational moment that arises when either the shaft offset $E$ or the axial offset $A_{1}$ are non-zero for the configuration in Figure 3. The vector $\boldsymbol{S}_{B}^{(B 2)}$ expresses the arm of the rotational moment and is defined as the vector that 
points from the origin of $S_{B}$ to the origin of $S_{2}$. The position and orientation of the corresponding surface normal for any point on the surface of the pinion w.r.t. the frame $S_{B}$ is given by:

$$
\begin{gathered}
\overline{\boldsymbol{r}}_{B}^{(1)}=\mathbf{T}_{B 1}\left(\phi_{z 1}\right) \overline{\boldsymbol{r}}_{1}^{(1)} \\
\boldsymbol{N}_{B}^{(1)}=\mathbf{L}_{B 1}\left(\phi_{z 1}\right) \boldsymbol{N}_{1}^{(1)}
\end{gathered}
$$

The vectors $\overline{\boldsymbol{r}}_{1}^{(1)}$ and $\overline{\boldsymbol{r}}_{B}^{(1)}$ are used to express the homogeneous form of the position vectors $\boldsymbol{r}_{1}^{(1)}$ and $\boldsymbol{r}_{B}^{(1)}$, respectively. The matrices $\mathbf{L}_{B 1}$ and $\mathbf{L}_{B 2}$ correspond to the rotational part of the homogeneous transformation matrices $\mathbf{T}_{B 1}$ and $\mathbf{T}_{B 2}$, respectively. The angular rotation vectors $\boldsymbol{\omega}_{B}^{(1)}$ of the pinion and $\boldsymbol{\omega}_{B}^{(2)}$ of the conjugate to the pinion are obtained by transforming the rotation about the gear rotational axis (local z-axis) to the Base frame, using $\mathbf{L}_{B 1}$ and $\mathbf{L}_{B 2}$ respectively. Their magnitudes $\omega^{(1)}$ and $\omega^{(2)}$ are defined through the gear ratio $m_{21}$. Equation (14) provides the transformation to compute the point on the conjugate flank in the local gear frame $S_{2}$, where $\overline{\boldsymbol{r}}_{1}^{(1)}$ and $\overline{\boldsymbol{r}}_{2}^{(2 c)}$ are the homogeneous forms of the position vectors $\boldsymbol{r}_{1}^{(1)}$ and $\boldsymbol{r}_{2}^{(2 c)}$, respectively.

$$
\overline{\boldsymbol{r}}_{2}^{(2 c)}=\mathbf{T}_{B 2}^{-1}\left(-m_{21} \phi_{z 1}\right) \mathbf{T}_{B 1}\left(\phi_{z 1}\right) \overline{\boldsymbol{r}}_{1}^{(1)}
$$

\subsubsection{Surface of roll angles and surface of action}

Solving the equation of meshing for one particular point $P$ on the tooth flank of the pinion yields a specific value of pinion roll angle $\phi_{z 1}$. The surface of roll angles $\mathcal{R}_{\phi}^{(1)}$ of the real pinion flank is the complete set of pinion roll angles that are obtained by solving Equation (11) for each point on the flank. The roll angle $\phi_{z 1}$ of a point on the pinion flank corresponds to the rotation that the pinion has to undergo for that point to reach its mating point on the conjugate flank. The location where this contact takes place is a point on the surface of action of the pinion flank. Similarly to the plane of action for involute cylindrical gears, the surface of action describes all the contact locations between two conjugate flanks. As shown in Figure 4 for the pinion, the pinion tooth flank will intersect its surface of action when it comes in contact with its conjugate for a given value of pinion roll angle $\phi_{z 1}$. Therefore it is possible to identify curves of contact points on the pinion tooth flank for which the two following contact conditions are satisfied: (i) coincidence of contact points (ii) and collinearity of surface normal vectors. Both conditions are expressed by Equations (15) and (16):

$$
\begin{aligned}
\boldsymbol{r}_{B}^{(1)} & =\boldsymbol{r}_{B}^{(2 c)} \\
\boldsymbol{N}_{B}^{(1)} & =-\boldsymbol{N}_{B}^{(2 c)}
\end{aligned}
$$

The position vector $\boldsymbol{r}_{B}^{(2 c)}$ is used to represent the points on the conjugate flank, while the vector $\boldsymbol{N}_{B}^{(2 c)}$ expresses the surface normal vector at $\boldsymbol{r}_{B}^{(2 c)}$.

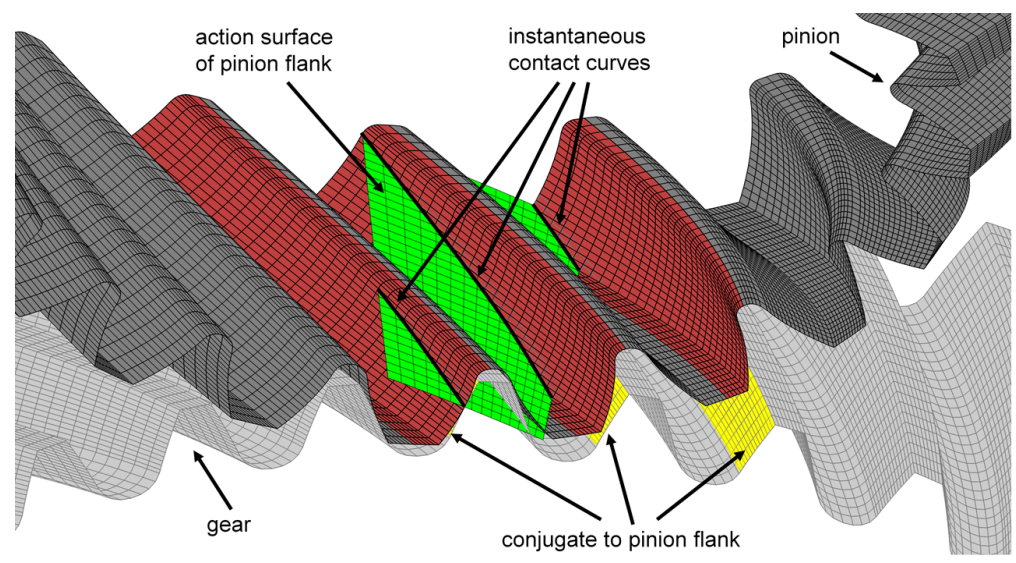

Figure 4: Example of the surface of action (green) of the left pinion flanks (red). 


\section{A multibody approach to tooth contact analysis}

A multibody (MB) approach to tooth contact analysis is proposed to develop a gear contact element that can be used for the simulation of both the static and dynamic contact behavior of spiral bevel gears. A study of the dynamic behavior is postponed to future work, as this paper will focus on the development and validation of the static behavior of the gear pair. To appropriately validate the proposed model LTCA is performed by constraining the multibody model in such a way that it can be easily compared in Section 5 with results that are obtained through nonlinear finite element contact simulations. In the presented model of Figure 6 both the gear and the pinion are modeled as rigid bodies that are connected to a ground body to remove their respective degrees of freedom (DOFs). The pinion is constrained in such a way that it can only rotate about its local z-axis, whereas the gear body is clamped to ground to remove its DOFs at each step of the simulation. The deflections of the gear teeth under load are taken into account by the contact force that acts between the meshing gear teeth.

The initial values that place both real gear flanks in contact at their conjugate point at the start of the simulation, are computed through use of the gear-based ease-off topography and the surface of roll angles for the pinion flank, using the methodology of [37] for a predefined gear geometry. A constant torque is then applied to the pinion in the global frame $S_{G}$ and the pinion's rotation is updated until static equilibrium is achieved. The arising contact forces are computed as the result of the gear tooth flank penetration, which is measured during the contact simulation by the developed contact detection algorithm. To compute the next step of the mesh cycle both pinion and gear are then indexed accordingly and the process is repeated. A schematic overview is given in Figure 5. The static transmission error (STE), given in Equation (17),

220 is computed at the end of each static simulation, based on the pinion rotation angle $\phi_{z 1}$ and gear rotation angle $\phi_{z 2}$.

$$
S T E=\left(\phi_{z 2}-\phi_{z 20}\right)-\frac{Z_{1}}{Z_{2}}\left(\phi_{z 1}-\phi_{z 10}\right)
$$

Here $\phi_{z 10}$ and $\phi_{z 20}$ correspond to the initial angular displacement of the pinion and the gear that are required to position the mating tooth flanks in contact at the initial conjugate point, while $Z_{1}$ and $Z_{2}$ correspond to the number of teeth for the pinon and gear, respectively [38].

\subsection{Efficient contact detection}

Similarly to cylindrical gears, where the (roll) angle of the involute is used to express the position of the contact points on the line/plane of action [39], the surface of roll angles can be used to accurately and efficiently compute the contact location on the surface of action for spiral bevel gears. To allow for numerically efficient contact detection within the proposed multibody framework a methodology, similar to the one available for cylindrical gears, is developed that includes the surface of roll angles of both the real pinion and the real gear. Using both surfaces of roll angles allows to determine the contact points on the real teeth flanks (both for the gear and the pinion) without the requirement of ease-off topography but rather through the penetration of the real flank geometry. This can prove highly relevant for a general case, in which the angular rotations of the gears are no longer prescribed by the kinematic relationship of the gear ratio but rather are the result of external forces and arising contact forces, e.g. misaligned conditions. The contact detection methodology is described considering only one type of flank pairs, i.e. either the right or the left flank of the tooth, since the process to include the other flank pairs is identical.

\subsubsection{Kinematics of the gear pair}

In the proposed methodology the position and orientation of both gears are described w.r.t. a global reference frame $S_{G}$. Any point on the pinion tooth surface, given by $\boldsymbol{r}_{1}^{(1)}$ in the pinion reference frame $S_{1}$, can be expressed in $S_{G}$ using the following transformation:

$$
\boldsymbol{r}_{G}^{(1)}=\boldsymbol{R}_{G}^{(1)}+\mathbf{A}_{1} \boldsymbol{r}_{1}^{(1)}
$$

The global position of the origin of the reference frame $S_{1}$ is given by the vector $\boldsymbol{R}_{G}^{(1)}$, while the rotation matrix $\mathbf{A}_{1}$ expresses the orientation of the axes of $S_{1}$ w.r.t. $S_{G}$ [40]. Similarly to Equation (18), any point 


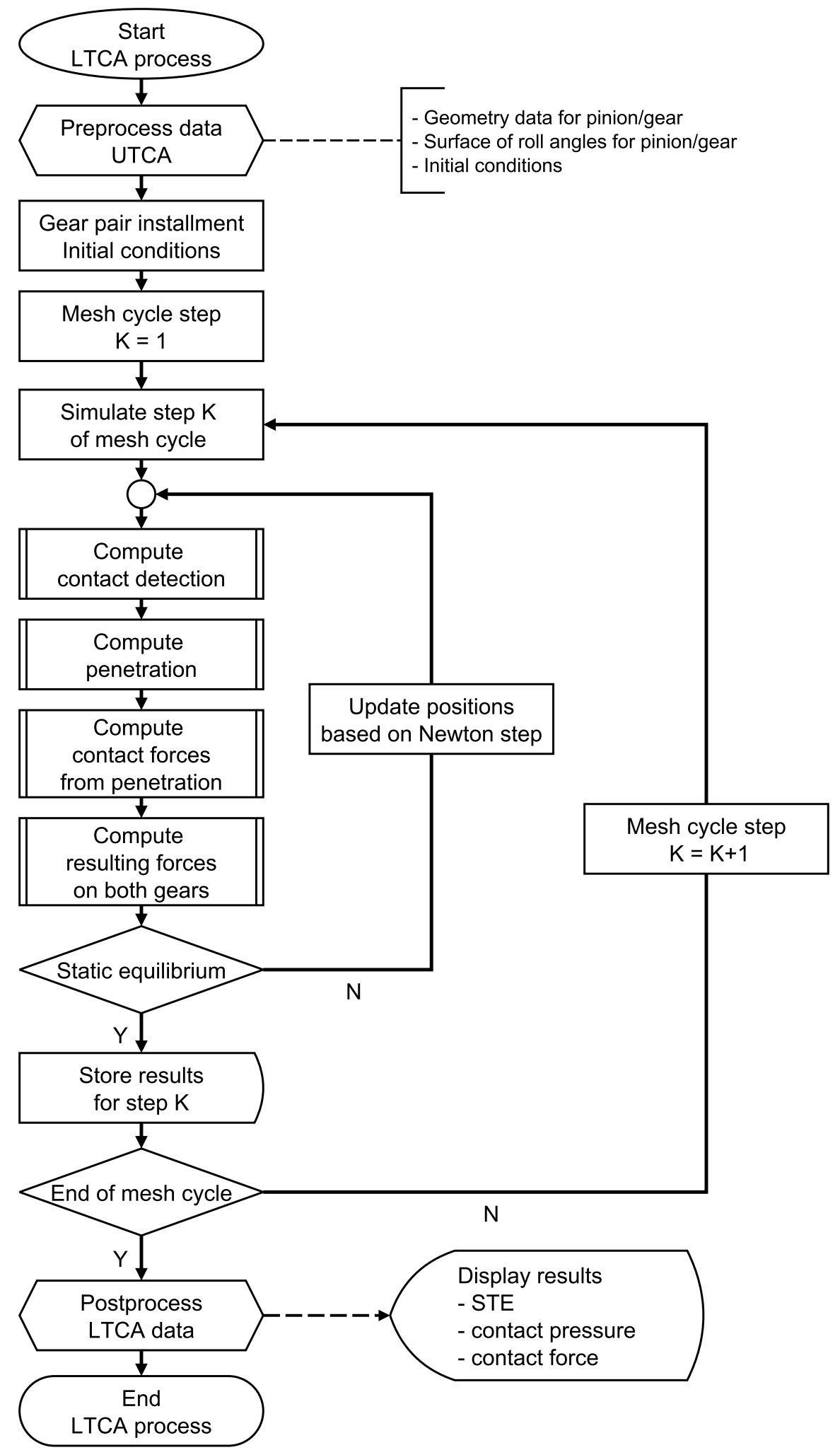

Figure 5: Overview of major steps of the loaded tooth contact analysis process. 


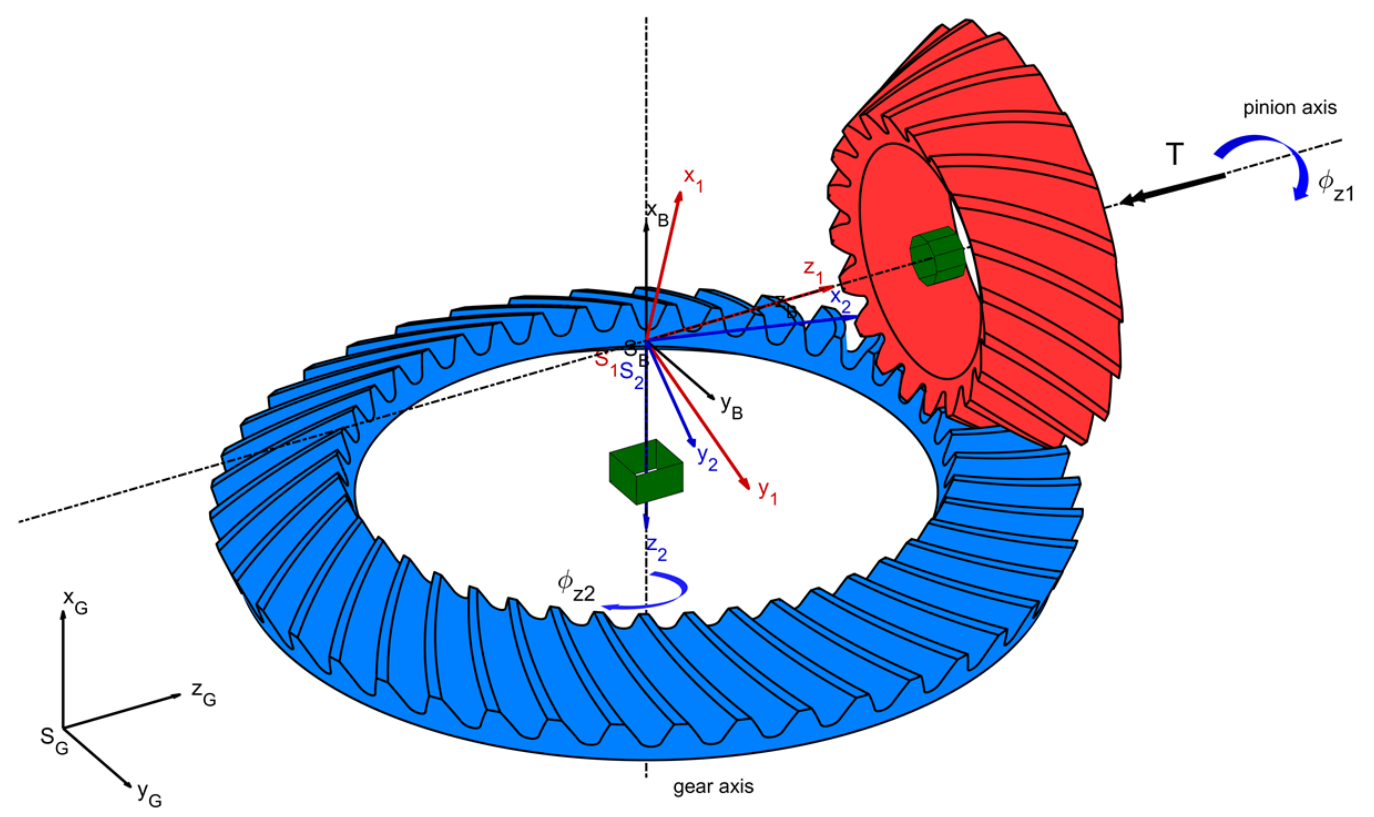

Figure 6: Model of the spiral bevel gear pair represented in the global reference frame $S_{G}$ for a given configuration of $E=0 \mathrm{~mm}$, $A_{1}=0 \mathrm{~mm}, A_{2}=0 \mathrm{~mm}, \gamma=-90 \mathrm{deg}$. The local reference frames $S_{1}$ and $S_{2}$ are used to describe the gear geometry.

on the gear tooth surface, given by $\boldsymbol{r}_{2}^{(2)}$ in the gear reference frame $S_{2}$, can be written in $S_{G}$ using:

$$
\boldsymbol{r}_{G}^{(2)}=\boldsymbol{R}_{G}^{(2)}+\mathbf{A}_{2} \boldsymbol{r}_{2}^{(2)}
$$
is then defined w.r.t. $S_{G}$ to describe the relative position and orientation of the gear pair. A set of Bryant angles $\left(\phi_{x}, \phi_{y}, \phi_{z}\right)$ is used to parameterize the rotational motion of the geared bodies w.r.t. $S_{B}$ [41]. To describe the configuration of Figure 6, the rotational convention $Y-X-Z$ is chosen, since it allows to describe the rotations about the $\mathrm{Y}$ - and Z-axis of $S_{B}$ in a range of $[0,2 \pi]$, while having its singularity for $\phi_{x}= \pm \pi / 2$.

250 Using the expressions in [42], the instantaneous rotation angles $\phi_{z 1}$ and $\phi_{z 2}$ for pinion and gear, respectively, are then computed based on the orientation of $S_{1}$ and $S_{2}$, respectively, w.r.t. $S_{B}$.

\subsubsection{Contact lines for multiple flank pairs}

Once the instantaneous rotational angles $\phi_{z 1}$ and $\phi_{z 2}$ for pinion and gear are known, their respective surfaces of roll angles $\mathcal{R}_{\phi}^{(1)}$ and $\mathcal{R}_{\phi}^{(2)}$ can be used to determine the potential contact curves on the real tooth flanks. In a loaded contact simulation different teeth pair can come into contact as the result of either the rotation of the gears or due to increased loading. An estimate for the number of teeth $N_{T}$ that are potentially in contact is determined based on the contact ratio $\epsilon_{\gamma}$ for spiral bevel gears and includes the profile contact ratio $\epsilon_{\alpha}$ and face contact ratio $\epsilon_{\beta}$ [43]. The calculation of the contact ratio involves the approximation of the spiral bevel gear pair as an equivalent spur gear pair that is seen from the back cone (Tredgold's approximation). Therefore the contact ratio only offers a rough approximation and a safety factor is introduced to overestimate $N_{T}$ as:

$$
N_{T}=2\left\lceil\epsilon_{\gamma}\right\rceil+1
$$

For each rotational configuration of the gear pair the $N_{T}$ closest tooth flank pairs are identified by computing the distance between each pinion flank and each gear flank, evaluated at the middle of the flank. Since all teeth of a gear are assumed to be identical, the roll surface of each flank $k$ of the gear pair can be computed based on the tooth number $T_{k}$ and the angular pitch $\tau$. Adopting a clock-wise tooth numbering 


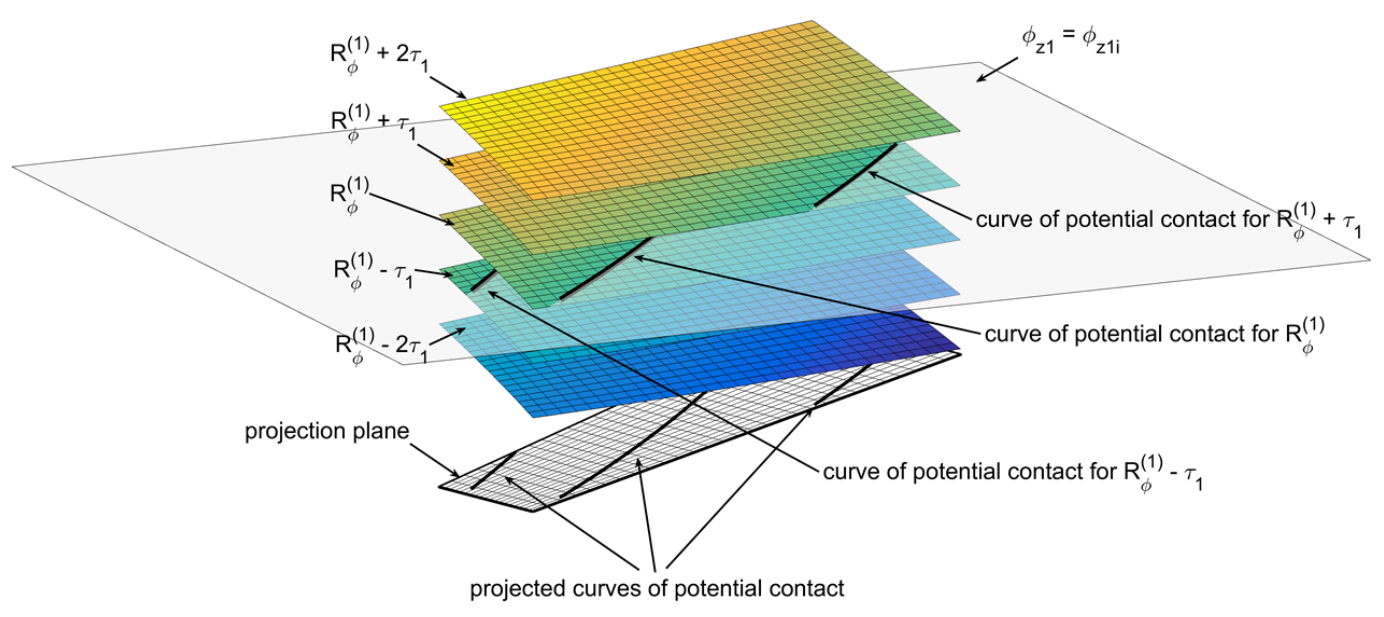

Figure 7: Curves of potential contact are created for different pinion flanks, starting from the reference roll surface $\mathcal{R}_{\phi}^{(1)}$. The contact curves are computed as the intersection of the surfaces of roll angle $\mathcal{R}_{\phi, k}^{(1)}$ with a plane of constant roll angle $\phi_{z 1}$.

when looking at the face cone of the spiral bevel gears, the roll surfaces for tooth pair $k$ are found by Equation (21) for the pinion and by Equation (22) for the gear.

$$
\begin{aligned}
\mathcal{R}_{\phi, k}^{(1)} & =\mathcal{R}_{\phi}^{(1)}-\left(T_{k}^{(1)}-1\right) \tau_{1} & \tau_{1} & =\frac{2 \pi}{Z_{1}} \\
\mathcal{R}_{\phi, k}^{(2)} & =\mathcal{R}_{\phi}^{(2)}-\left(T_{k}^{(2)}-1\right) \tau_{2} & \tau_{2} & =\frac{2 \pi}{Z_{2}}
\end{aligned}
$$

As illustrated in Figure 7 for the pinion element, multiple potential contact curves can then be created onto the gear-based projection plane as the intersection of a plane with constant roll angle $\phi_{z}$ and the surfaces of roll angle $\mathcal{R}_{\phi, k}$. Since $N_{T}$ is defined to be an overestimation of the number of teeth in contact, it is more than likely that a curve of potential contact points cannot be created for certain flank pairs $k$. In Figure 7 these flanks are the ones that have roll surfaces $\mathcal{R}_{\phi}^{(1)}-2 \tau_{1}$ and $\mathcal{R}_{\phi}^{(1)}+2 \tau_{1}$. The same procedure can be established for the gear element, using the gear's surface of roll angles $\mathcal{R}_{\phi}^{(2)}$ and the gear's rotational angle $\phi_{z 2}$.

For a general mismatched flank pair (non-conjugate) the use of two surfaces of roll angles, one for each of the real flanks, results into two curves of potential contact points, which do not coincide when they are projected onto the projection plane, as illustrated in Figure 8. Kolivand [11] proposed to replace the contact line of the real gear with the one of the conjugate to the real pinion when solving for the conditions of compatibility and equilibrium, since it would change the orientation and shape of the contact curve very little due to the high level of conformity between the mating flanks. In that case contact is determined based on the pinion's roll surface and the gear-based ease-off topography. Since in this work the contact forces are computed from the measured penetration between the real tooth flanks, a new contact curve is computed as an average of both contact curves by dividing the flank overlap into a finite number of slices and by computing maximum one actual contact point for each of the contacting tooth slices. Such an extension fits well within the philosophy of the general multibody approach where the behavior of the system is influenced by each individual body (i.e. gear). It also incorporates effects of both real surfaces in mesh and provides a better approximation of the contact curve in regions with low conformity. Additionally, it also enables to draw a natural analogy to a commonly used contact detection methodology for cylindrical gears that computes the penetration between the meshing involute flanks via the concept of roll angle of the involute in order to express the position of the tooth flank along the line/plane of action for each gear [44]. Therefore, illustrating how the proposed methodology generalizes a well-known contact detection method for cylindrical gears. 


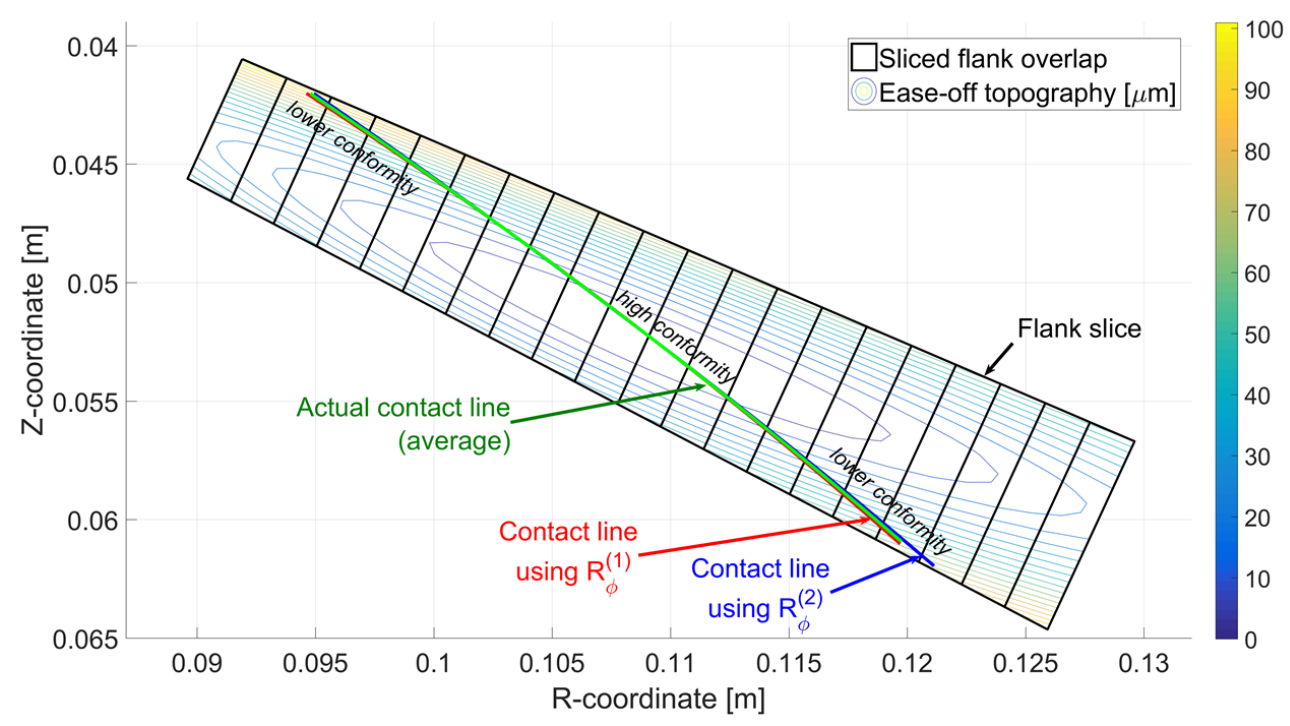

Figure 8: Actual contact curve (green) computed as the average of the contact curves, determined with roll surfaces $\mathcal{R}_{\phi}^{(1)}$ (red) and $\mathcal{R}_{\phi}^{(2)}$ (blue). The ease-off contours are added to indicate zones of high conformity.

\subsubsection{Flank penetration}

For each of the actual contact points (2D) the corresponding pinion and gear points (3D) are computed in order to determine the penetration between the mating flanks. The flank slice penetration $\delta$ is then computed by first calculating the distance between the point in the pinion flank $\boldsymbol{r}_{G}^{(1)}$ and the corresponding point on the gear flank $\boldsymbol{r}_{G}^{(2)}$, both expressed in the global reference frame $S_{G}$. The distance between the flank slices is then projected onto the local contact normal $\boldsymbol{N}_{G}^{c o n t}$ to obtain the flank slice penetration.

$$
\begin{aligned}
\delta & =\left(\boldsymbol{r}_{G}^{(1)}-\boldsymbol{r}_{G}^{(2)}\right) \cdot \boldsymbol{N}_{G}^{c o n t} \\
\boldsymbol{r}_{G}^{c o n t} & =\frac{1}{2}\left(\boldsymbol{r}_{G}^{(1)}+\boldsymbol{r}_{G}^{(2)}\right) \\
\boldsymbol{N}_{G}^{\text {cont }} & =\frac{1}{2}\left(\boldsymbol{N}_{G}^{(1)}-\boldsymbol{N}_{G}^{(2)}\right)
\end{aligned}
$$

The contact location $\boldsymbol{r}_{G}^{c o n t}$ is defined in the global frame $S_{G}$ as the average of the contact point on the pinion and on the gear. The direction of the contact normal $\boldsymbol{N}_{G}^{\text {cont }}$ is computed in the global frame $S_{G}$ as the average of the directions of the respective surface normal vectors $\boldsymbol{N}_{G}^{(1)}$ and $\boldsymbol{N}_{G}^{(2)}$, given by Equation (26) and Equation (27). The surface normal vectors are evaluated at the contact points on the respective flanks.

$$
\begin{aligned}
& \boldsymbol{N}_{G}^{(1)}=\mathbf{A}_{1} \boldsymbol{N}_{1}^{(1)} \\
& \boldsymbol{N}_{G}^{(2)}=\mathbf{A}_{2} \boldsymbol{N}_{2}^{(2)}
\end{aligned}
$$

Since both flanks are penetrating their respective contact points will not be coincident and their surface normal will not be collinear. Although such deviations are expected to be small, the averaging is still performed to compute a common contact point and contact direction along which the contact force can be applied to both gears.

\subsection{Tooth compliance modeling}

To compute the contact forces that act on the different tooth slices, it is assumed that the penetration between the two rigid tooth surfaces is identical to the deformation that these surfaces would experience 


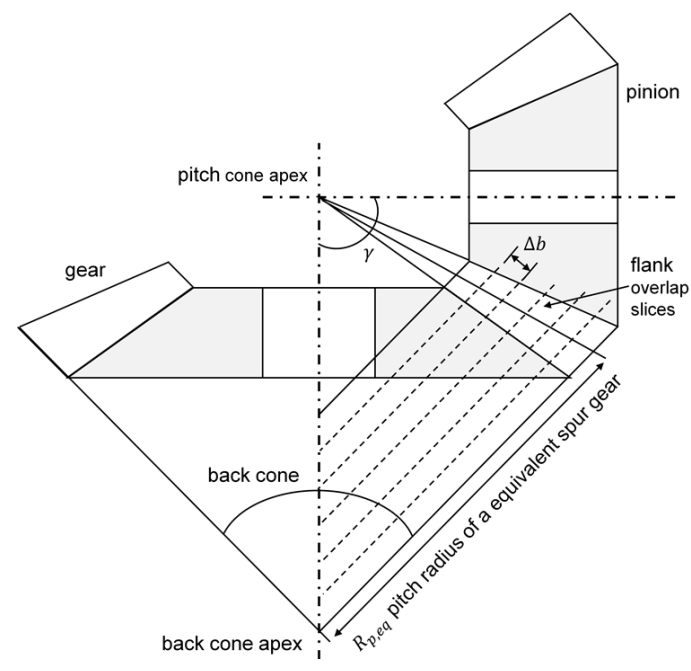

(a) Tredgold's approximation

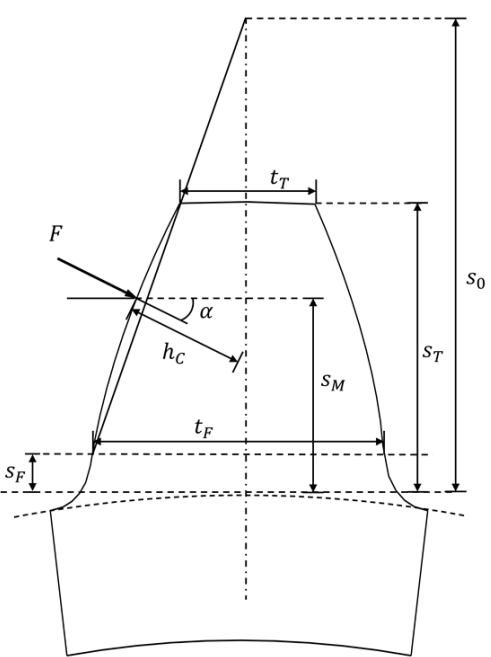

(b) Tooth slice in normal plane

Figure 9: To compute the global compliance components each tooth slice is approximated in the transverse plane as an equivalent spur gear (Tredgold's approximation), for which the tooth dimension are projected onto the local normal plane.

if they were flexible. Based on the idea of Andersson and Vedmar [45], the deformation between the contacting tooth slices is modeled as the result of two contributions; (i) a local contribution that describes the deformation in the region of contact between the two bodies, and (ii) a set of global contributions that describe the deflection of the individual teeth outside the contact zone. In the presented model each of the contributions is modeled in an analytical fashion.

\subsubsection{Global tooth compliance}

To obtain an approximation for the global compliance of a single gear tooth, Tredgold's approximation is applied to each of the tooth slices for which penetration is detected. Tredgold's approximation, as shown in Figure 9a, approximates the bevel gear pair as an equivalent spur gear pair when seen from the back cone. Each tooth segment is therefore approximated by an equivalent involute tooth in the normal plane and the formulas, derived by Nakada and Utagawa [29], are used to compute the global tooth compliance. The bending $\delta_{b}$, shear $\delta_{s}$ and gear body foundation $\delta_{f}$ deformation contributions are computed by approximating each tooth slice of width $\Delta b$ as a beam with variable thickness. The resulting global deformation $\delta_{g}$ at the contact point of the segment is found as the sum of the individual contributions:

$$
\begin{aligned}
\delta_{g}=\delta_{b}+\delta_{s}+\delta_{f} \\
\delta_{b}=\frac{12 F s_{F} \cos ^{2}(\alpha)}{E \Delta b t_{F}^{3}}\left[s_{M}+\frac{s_{F}^{3}}{3}-s_{F} s_{M}\right] \\
+\frac{6 F\left(s_{0}-s_{F}\right)^{3} \cos ^{2}(\alpha)}{E \Delta b t_{F}^{3}}\left[\frac{s_{0}-s_{M}}{s_{0}-s_{F}}\left(4-\frac{s_{0}-s_{M}}{s_{0}-s_{F}}\right)\right. \\
\left.\quad-2 \ln \left(\frac{s_{0}-s_{M}}{s_{0}-s_{F}}\right)-3\right] \\
\delta_{s}=\frac{2(1+\nu) F \cos ^{2}(\alpha)}{E \Delta b t_{F}}\left[s_{F}+\left(s_{0}-s_{F}\right) \ln \left(\frac{s_{0}-s_{F}}{s_{0}-s_{M}}\right)\right] \\
\delta_{f}=\frac{24 F s_{M}^{2} \cos ^{2}(\alpha)}{\pi E \Delta b t_{F}^{2}} \\
s_{0}=\frac{s_{T} t_{F}-s_{F} t_{T}}{t_{F}-t_{T}}
\end{aligned}
$$


To match the direction of the contact force, given by the direction of the contact normal $\boldsymbol{N}_{G}^{c o n t}$, the deformation components are computed in a plane that is normal to the tooth surface at the contact point. The cross-section of the tooth as seen in this normal plane is provided in Figure 9b. The tooth thickness in the normal plane at the bottom $t_{F}$ and top $t_{T}$ of the active flank are computed by projecting the transverse tooth thickness values of the equivalent spur gear onto the normal plane. The transverse tooth thicknesses for the equivalent spur gear are computed based on the actual tooth geometry. The tooth thicknesses are computed at the front and back of the bevel gear tooth and then assumed to vary linearly along the face width. The tooth height values $s_{F}, s_{T}, s_{0}$ at, respectively, the bottom and top of the active flank and the intersection with the middle of the tooth are directly computed from the tooth geometry at the contact point.

\subsubsection{Local contact compliance}

For a general case of mismatched flanks the instantaneous contact between two meshing flanks can be described as a point contact under no-load conditions. When load is applied this point contact will spread into a line contact that runs over a part of the tooth flanks. A Hertzian line contact, oriented along the direction of the contact curve, is assumed for each of the contacting tooth slices. The nonlinear contact deformation $\delta_{c}$ that arises from the load $F$ that is applied onto the contact segment of length $l$ can be described with a closed-form formula that was derived by Weber and Banaschek [46].

$$
\delta_{c}=\frac{F}{\pi l}\left(\frac{1-\nu_{1}^{2}}{E_{1}}+\frac{1-\nu_{2}^{2}}{E_{2}}\right)\left[\ln \left(\frac{4 h_{C 1} h_{C 2}}{a^{2}}\right)-\frac{1}{2}\left(\frac{\nu_{1}}{1-\nu_{1}}+\frac{\nu_{2}}{1-\nu_{2}}\right)\right]
$$

Equation (33) was derived under the assumption that in the near proximity of the contact the two contacting flanks can be well represented as cylinders with radii of curvature $r_{1}$ and $r_{2}$, as illustrated in Figure 10 . The parameters $h_{C 1}$ and $h_{C 2}$ correspond to the distance from the point of contact to the center of the tooth slice for the pinion and the gear, respectively. An example for a general $h_{C}$ is given in Figure $9 \mathrm{~b}$. Hertz derived the analytical formulas that allow to compute the half contact width $a$ and the maximum contact pressure $p_{0}$ as functions of a contact load $F[47]$.

$$
\begin{aligned}
a & =\left[\frac{4 F}{\pi l} \rho^{*}\left(\frac{1-\nu_{1}^{2}}{E_{1}}+\frac{1-\nu_{2}^{2}}{E_{2}}\right)\right]^{1 / 2} \\
p_{0} & =\frac{2 F}{\pi a l}
\end{aligned}
$$

The material characteristics are taken into account through the Young's modulus $E_{1}$ and $E_{2}$ and the Poisson's ratios $\nu_{1}$ and $\nu_{2}$ of the pinion and of the gear, respectively. The equivalent radius of curvature $\rho^{*}$ accounts for the relative curvature of the two cylinders at the contact point. The normal curvatures $\kappa_{n 1}$ and $\kappa_{n 2}$ in Equation (36) are obtained for each contact points through Euler's curvature formula of Equation (4).

$$
\frac{1}{\rho^{*}}=\frac{1}{r_{1}}+\frac{1}{r_{2}}=\kappa_{n 1}+\kappa_{n 2}
$$

\subsubsection{Computation of the gear contact forces}

The contact forces for pinion and gear are computed as the result of the individual contact forces that are acting between the different tooth segments. The total deformation of two contacting flank segments is found as the combination of the global deformation of the pinion $\delta_{g}^{(1)}$, the global deformation of the gear $\delta_{g}^{(2)}$ and the contact deformation $\delta_{c}$. Considering that at a given moment during the simulation $N_{T}$ tooth pairs are possibly in contact (i.e. penetrating or not) and that each flank of the tooth pair is discretized into $N_{S}$ segments, the total deformation $\delta_{k i}\left(F_{k i}\right)$ for a single flank pair segment $i$ of tooth pair $k$ becomes:

$$
\delta_{k i}\left(F_{k i}\right)=\delta_{g, k i}^{(1)}\left(F_{k i}\right)+\delta_{g, k i}^{(2)}\left(F_{k i}\right)+\delta_{c, k i}\left(F_{k i}\right)
$$




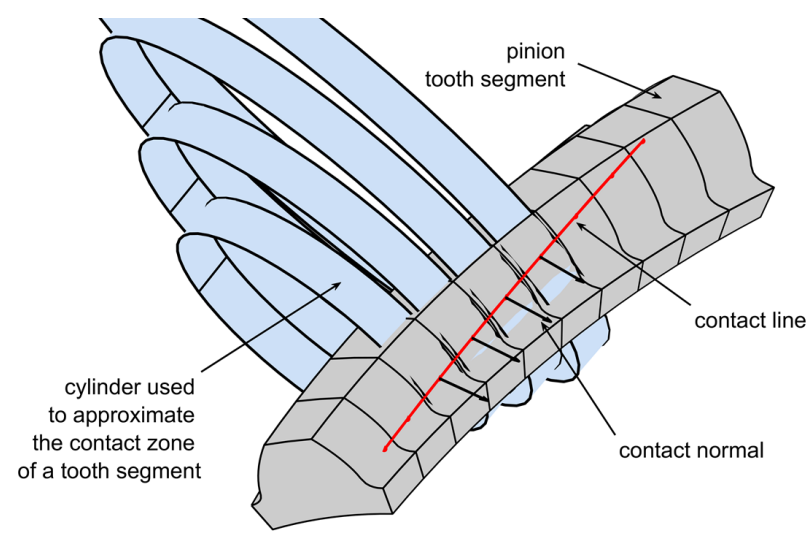

Figure 10: A Hertzian line contact is adopted to describe the contact deformation of each pair of tooth segments. The line contact is oriented along the contact direction, while the surfaces are locally approximated as cylinders.

The contact force $F_{k i}$ that acts on the flank pair segment is found by assuming that the total deformation $\delta_{k i}$ is the same as the penetration that is computed by Equation (23) under the assumption that contacting flanks are rigid surfaces. If no penetration between the flank segments is detected, the contact force $F_{k i}$ for that segment pair is considered to be zero, otherwise the contact is computed by numerically inverting Equation (37). The resulting contact forces $\boldsymbol{F}_{c}^{(1)}$ and $\boldsymbol{F}_{c}^{(2)}$ that act on the pinion and gear body, respectively, are found as the result of the individual contact forces magnitudes $F_{k i}$ of each segment.

\begin{tabular}{lcc}
\hline Blank Data & Pinion & Gear \\
\hline Number of teeth & 20 & 43 \\
Diametral pitch [1/mm] & \multicolumn{2}{c}{0.1676} \\
Shaft angle [deg.] & \multicolumn{2}{c}{90.0000} \\
Face width [mm] & \multicolumn{2}{c}{41.0000} \\
Mean cone distance [mm] & \multicolumn{2}{c}{120.9400} \\
Mean spiral angle [deg.] & \multicolumn{2}{c}{32.0000} \\
Hand of spiral [-] & RH & LH \\
Pitch Angle [deg.] & 24.9439 & 65.0561 \\
Root Angle [deg.] & 23.1666 & 61.8166 \\
Face Angle [deg.] & 28.1833 & 66.8333 \\
Outer addendum [mm] & 6.8900 & 3.2500 \\
Outer dedendum [mm] & 4.3700 & 8.0100 \\
Young's modulus [GPa] & 210.00 & 210.00 \\
Poisson's ratio [-] & 0.30 & 0.30 \\
\hline
\end{tabular}

Table 1: Blank data for the spiral bevel gear pair.

\section{Results and validation}

To demonstrate its capabilities, the proposed methodology is applied to the analysis of a spiral bevel gear set, whose blank data is listed in Table 1 and whose geometry is visualized in Figure 6 . The gear pair data was taken from literature [2] in order to have a first level validation for both the geometry and UTCA results during the development process. The analysis focuses on the contact between the concave pinion flanks and the convex gear flanks, which corresponds to the flanks on the right hand-side of the respective gear teeth when looking from the face cone. The results for transmission error, contact curves and contact pressure that were obtained with the proposed methodology, are validated against results that were obtained with NX Nastran (SOL 601), a commercial software package for nonlinear finite element simulations [48]. 


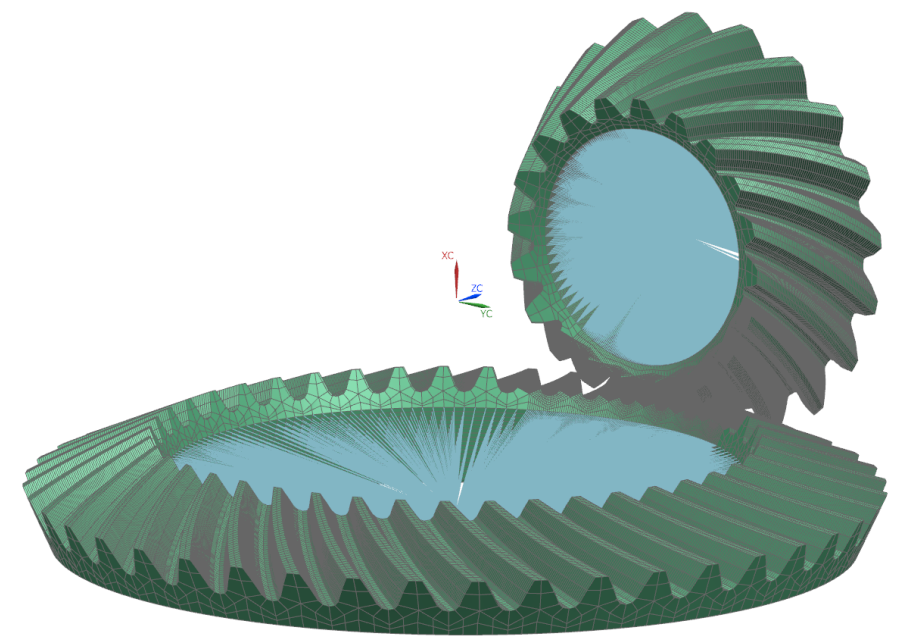

Figure 11: Finite element model of the spiral bevel gear pair, used to validate the proposed methodology through nonlinear finite element contact simulations.

\begin{tabular}{lccccc}
\hline Number of Elements [el.] & total & 1 tooth (contact) & active profile & root profile & face width \\
\hline Pinion (Base) & $242250 \mathrm{el}$. & $36750 \mathrm{el}$. & $35 \mathrm{el}$. & $10 \mathrm{el}$. & $75 \mathrm{el}$. \\
Gear (Base) & $269850 \mathrm{el}$. & $36750 \mathrm{el}$. & $35 \mathrm{el}$. & $10 \mathrm{el}$. & $75 \mathrm{el}$. \\
\hline Pinion (Refined) & $329760 \mathrm{el}$. & $50400 \mathrm{el}$ & $42 \mathrm{el}$ & $10 \mathrm{el}$. & $90 \mathrm{el}$. \\
Gear (Refined) & $362880 \mathrm{el}$. & $50400 \mathrm{el}$. & $42 \mathrm{el}$. & $10 \mathrm{el}$. & $90 \mathrm{el}$. \\
\hline
\end{tabular}

Table 2: Overview of the number of elements (el.) that were used for the complete model and the contacting teeth specifically for the base and refined FE model (same for gear and pinion).

\subsection{Finite element model creation}

A finite element (FE) model of the gear pair is created to serve as a reference model during the validation of the proposed methodology. Both the pinion and gear geometry (blank and teeth) are discretized as a connected set of 3D elements (HEXA8) for which the tooth flank nodes are directly computed by the simulation of the manufacturing process (Figure 1). The contact detection is achieved through discretization of the contact interfaces for which the so-called node-to-element method is used [49].

To reduce the overall size of the FE models and thus the required computational time, the FE model of each gear is created by combining three different sections, each with its own mesh density. A finely meshed section includes the teeth that are considered to be in contact. The coarsely meshed section is made up of the non-contacting teeth that are still modeled to correctly describe the stiffness of the gear body, while a third section contains intermediately meshed teeth that hold the fine and coarse sections together. The resulting FE model is presented in Figure 11.

Different models were created to study mesh convergence. An overview of the number of elements that was used to create the FE model of the pinion and the gear are provided in Table 2 for both the Base and Refined model. The table also includes the number of elements along each direction of a finely meshed tooth (contact) as well as the total number of elements for a single tooth. Based on [2], the number of elements for the teeth in contact were chosen. However, at the end of a mesh size convergence study this number was nearly doubled for the Base model and more than doubled for the Refined model. To verify mesh size convergence for the Base model, the Refined model was created and both transmission error (Figure 12) and overall contact pattern and contact pressure (Figure 13) were analyzed. Each static simulation was computed by applying the specified load to the pinion, while the gear element was kept fixed. Since less deformation occurs, the effect of mesh discretization is more pronounced for lower loads. Therefore, the mesh convergence study was performed for two load cases: $0.01 \mathrm{Nm}$ and $200 \mathrm{Nm}$. A load of $0.01 \mathrm{Nm}$ was applied 
to the pinion to approximate unloaded conditions, while a load of $200 \mathrm{Nm}$ represents a moderately low load for which a clearly developed contact pattern can be identified. Comparing the STE curves of Figures 12a and $12 \mathrm{~b}$ together with the overall contact patterns of Figures $13 \mathrm{a}$ and $13 \mathrm{~b}$, it can be concluded that both the Base and the Refined model produce similar results and that existing differences are small. Thus, allowing the Base model to be used as a good reference model for validation of the presented methodology.

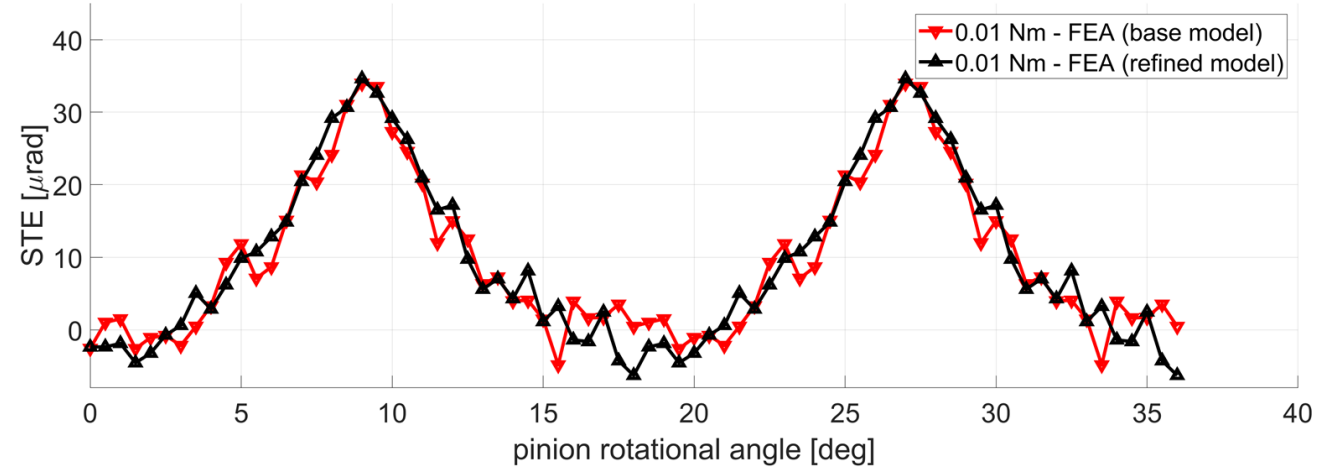

(a) Static transmission error for $0.01 \mathrm{Nm}$ (unloaded).

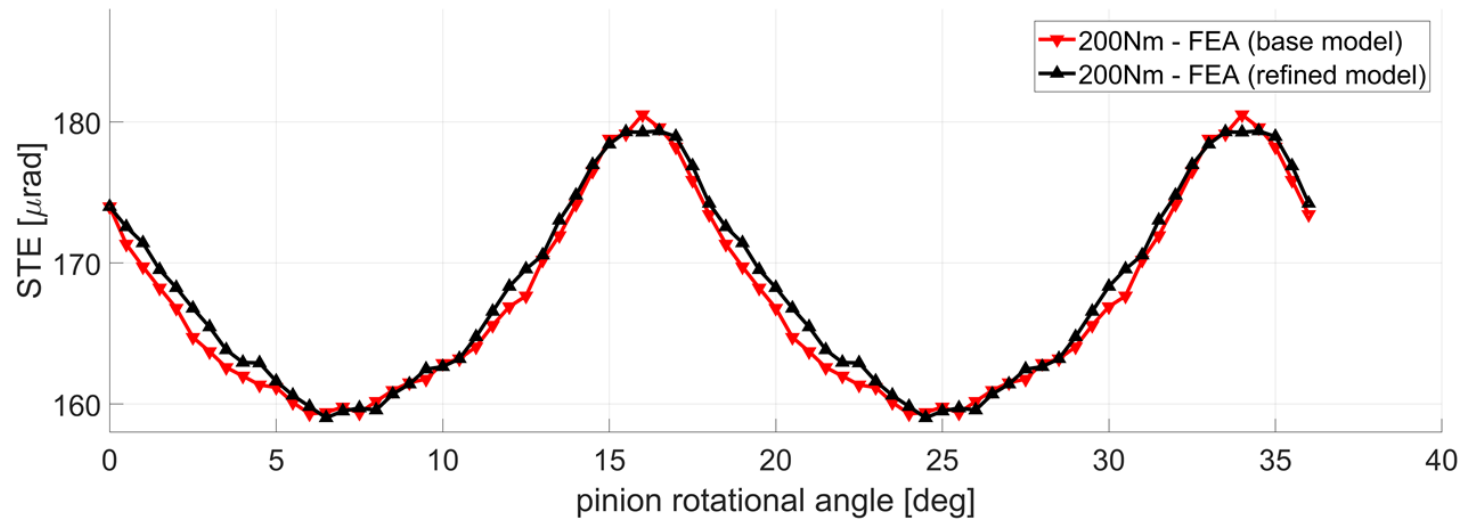

(b) Static transmission error for $200 \mathrm{Nm}$

Figure 12: Mesh Convergence - Comparison of the transmission error for Base and Refined model for $0.01 \mathrm{Nm}$ and $200 \mathrm{Nm}$.

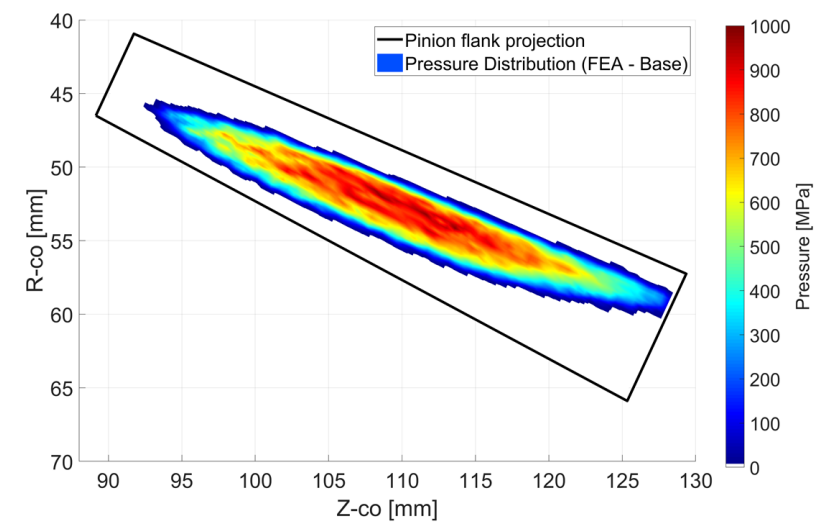

(a) Overall contact pattern for Base model at $200 \mathrm{Nm}$.

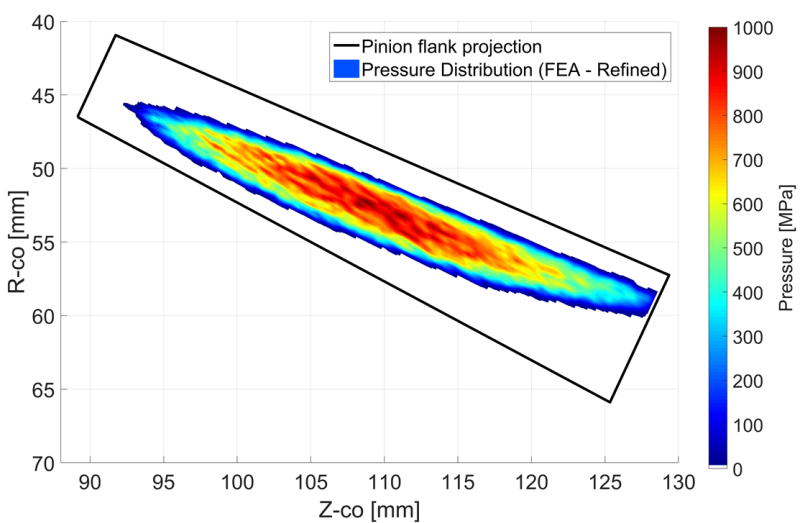

(b) Overall contact pattern for Refined model at $200 \mathrm{Nm}$.

Figure 13: Mesh Convergence - Comparison of the overall contact pattern at $200 \mathrm{Nm}$ for Base and Refined model. 


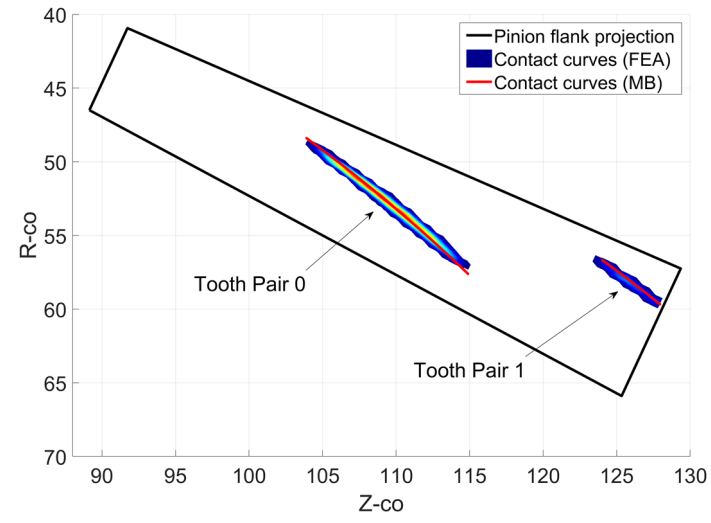

(a) Comparison of the instantaneous contact curves for $200 \mathrm{Nm}$.

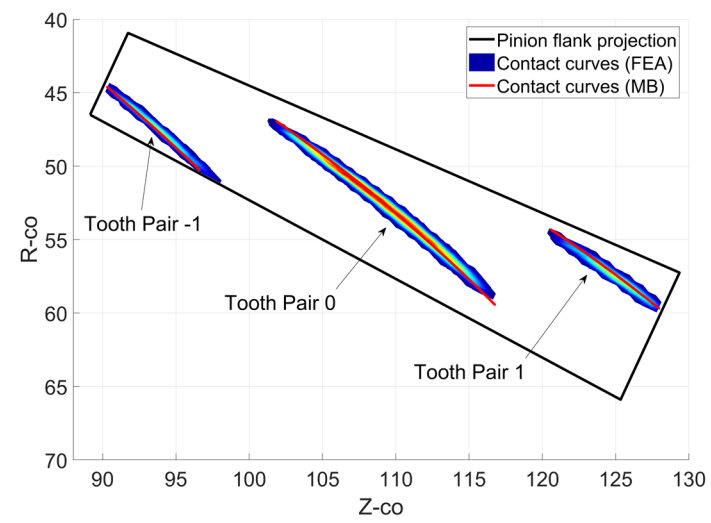

(b) Comparison of the instantaneous contact curves for $700 \mathrm{Nm}$.

Figure 14: Comparison of instantaneous contact curves for $200 \mathrm{Nm}$ and $700 \mathrm{Nm}$. The contact points of all the flank pairs in contact are projected onto a common flank projection for the pinion element. The tooth flank pair that carries the majority of the load is given index 0 , while the neighboring left and right flank pairs are given index -1 and 1 , respectively. single tooth flank pair will be in contact for unloaded and lightly loaded conditions. As the load increases, the occurring deformation will cause multiple tooth flanks to come into contact. Figure 14a and Figure 14b show that for this specific gear pair two and three tooth flank pairs carry the contact for a given load level of $200 \mathrm{Nm}$ and $700 \mathrm{Nm}$, respectively. In order to visualize the results for different tooth flank pairs the contact points of the flank pairs in contact are projected onto a common flank projection for the pinion. The tooth flank that carries the majority of the load is identified through index 0 , while the neighboring left and right flank pairs are given index -1 and 1, respectively. The obtained results show that a good correlation in terms of instantaneous contact location is obtained between the reference FE model and the developed analytical model for loaded contact conditions.

The overall loaded contact pattern provides a natural way of assessing the resulting loaded region, since it can be observed in loaded experiments. It can be created by keeping track of all the instantaneous contact curves that occur through a complete mesh cycle. Figure 15 provides a comparison of the loaded contact pattern for three different load cases: $200 \mathrm{Nm}, 500 \mathrm{Nm}$ and $700 \mathrm{Nm}$. To allow for a direct one-to-one comparison of both models the maximum contact pressure during the mesh cycles is displayed on a projection of the pinion flank. The nodal contact pressure is computed on the nodes of the slave surfaces (pinion) during the nonlinear FE contact simulation, while the contact pressure for the analytical model is computed using

405 Equation (35). Figures 15a, 15c and 15e show the overall contact pressure distribution and the boundary of the contact pattern, which are computed with the proposed analytical model for the respective load cases. Figures $15 \mathrm{~b}, 15 \mathrm{~d}$ and $15 \mathrm{f}$ in their turn illustrate the overall contact pressure distribution that was obtained through nonlinear FEA simulations for the same load cases. The contact pattern boundary of the analytical model is also displayed on these figures to facilitate the comparison of the size of the contact patterns. Obtained results in terms of overall loaded contact pattern for the analytical model show a remarkably good correlation in terms of actual contact region and maximum contact pressure, when compared to the FEA results. While the contact pressure distribution for the FE results is accurately represented, it does 
show that the effect of mesh discretization is more pronounced for lower load cases (e.g. $200 \mathrm{Nm}$ ). The analytical model on the other hand does not suffer from such effects, since the local contact compliance of Equation (33) assumes the Hertzian pressure distribution for a line load for each of the contacting tooth slices.

Figure 16 allows to compare the static transmission error curves in terms of shape and peak-to-peak value for all the considered load cases. Figures $16 \mathrm{a}$ and $16 \mathrm{~b}$ are ordered in such a way that they facilitate easy reading of the results. Overall the STE curves, obtained with the proposed model, show a good correlation for both shape and peak-to-peak value. Similar to cylindrical gears, micro-modifications (ease-off) is also applied to bevel gear flank to counteract the deformation due to loading. Based on the obtained results the designed operating load, where the micro-modifications balance out the occurring deformation and thus minimize the peak-to-peak TE, appears to be located around $100 \mathrm{Nm}$. Figure 16b shows that for the lower torque ranges the STE is very well predicted, which is a good indicator for the correctness of the contact detection and the local contact compliance model. As the load increases and the teeth start to deform more, differences arise. Such differences are not unexpected since Tredgold's approximation and the global compliance formulas of Equations (28) to (31) do introduce some approximations into the model. The effect of load sharing, due to the coupled deformation of the neighboring teeth, is not yet included into the proposed model. Since effects of load sharing become more pronounced with an increase of the load, they can most likely be connected with the noticeable increase in phase difference between the STE curves of both models for high loading conditions, as shown in Figure 16a.

To conclude a brief overview of the required computational time for both models is provided in Table 3 . To obtain an objective benchmark the proposed model was executed on the desktop computer (i7 @ $3.20 \mathrm{GHz}$ with 32 GB RAM) that was used to perform the nonlinear FE simulations. The differences in simulation time between both models can be contributed to the methodology for contact detection that was used, and the number of DOFs of each model. The element size that is required to accurately model the contacting tooth geometry not only results in a higher number of elements that have to be included into the general contact search but also increases the overall size of the problem (number of DOFs). The proposed approach benefits from an optimized contact search, which is based on pre-computed surfaces of roll angles, and a lower number of DOFs due to a contact force computation that is based on the penetration of rigid surfaces. 


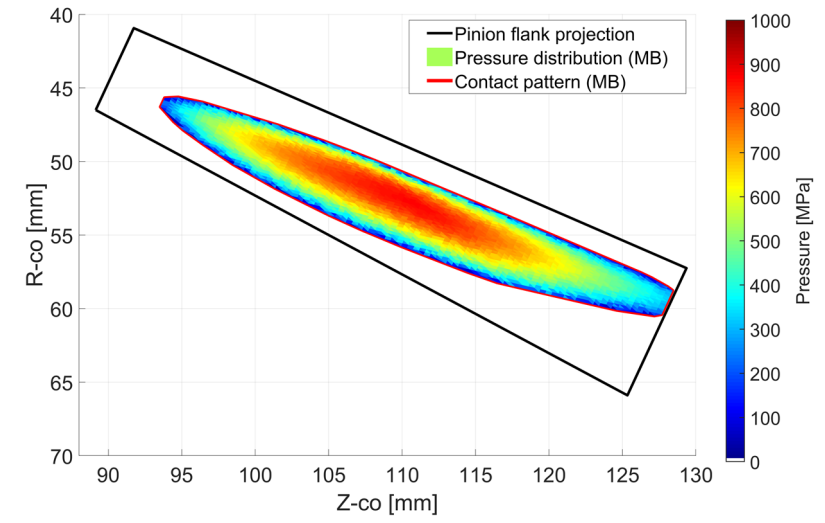

(a) Contact pattern, predicted by analytical model, for $200 \mathrm{Nm}$.

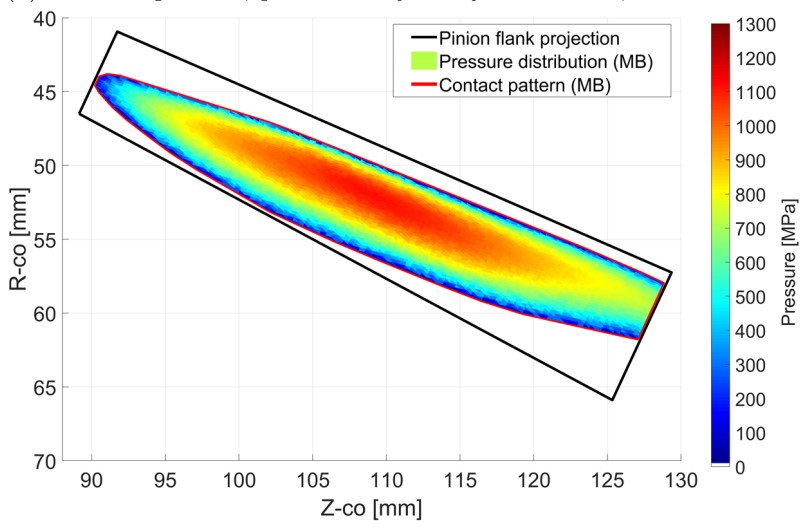

(c) Contact pattern, predicted by analytical model, for $500 \mathrm{Nm}$.

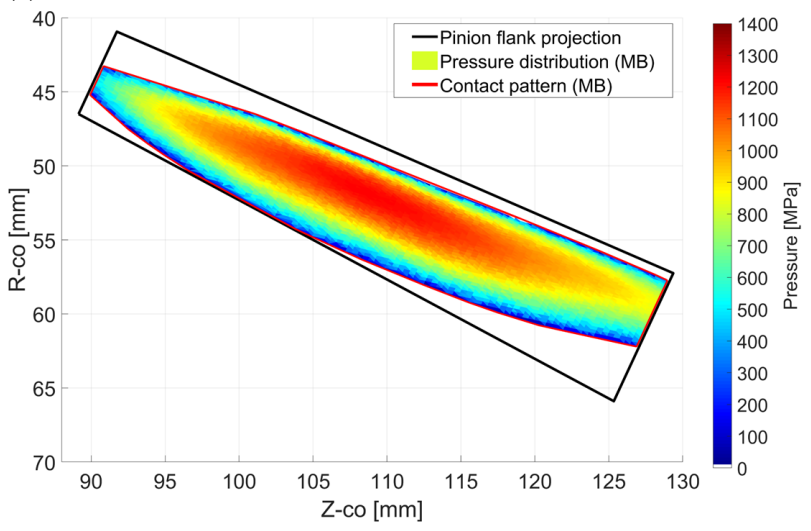

(e) Contact pattern, predicted by analytical model, for $700 \mathrm{Nm}$.

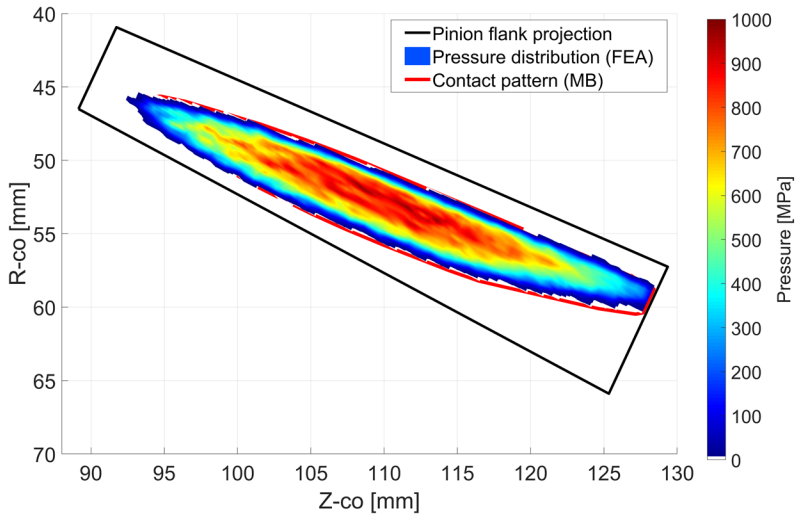

(b) Contact pattern, computed by nonlinear FEA, for $200 \mathrm{Nm}$.

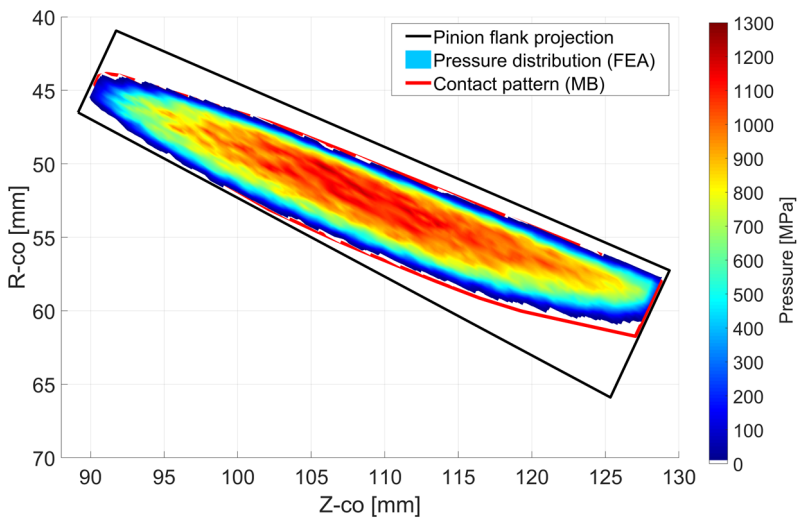

(d) Contact pattern, computed by nonlinear FEA, for $500 \mathrm{Nm}$.

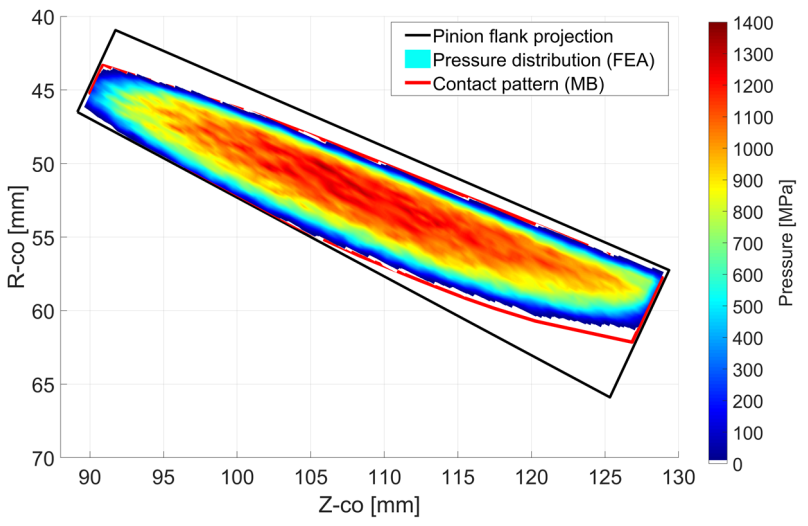

(f) Contact pattern, computed by nonlinear FEA, for $700 \mathrm{Nm}$.

Figure 15: Comparison of the overall contact pattern and the contact pressure distribution for $200 \mathrm{Nm}, 500 \mathrm{Nm}$ and $700 \mathrm{Nm}$. The results are projected onto the pinion flank.

\begin{tabular}{lccc}
\hline Computation time & FEA Model & MB Model (50 slices) & MB Model (100 slices) \\
\hline Single mesh cycle step & $10,625 \mathrm{~s}$ & $1.19 \mathrm{~s}$ & $1.39 \mathrm{~s}$ \\
Full mesh cycle (37 steps) & $4 \mathrm{~d} 13 \mathrm{~h} 12 \mathrm{~m} 05 \mathrm{~s}$ & $44.12 \mathrm{~s}$ & $51.58 \mathrm{~s}$ \\
\hline
\end{tabular}

Table 3: Overview of the computation time for a single step and full mesh cycle for the nonlinear FE model (Base model) and the analytical model for 50 and 100 flank overlap slices, respectively. 


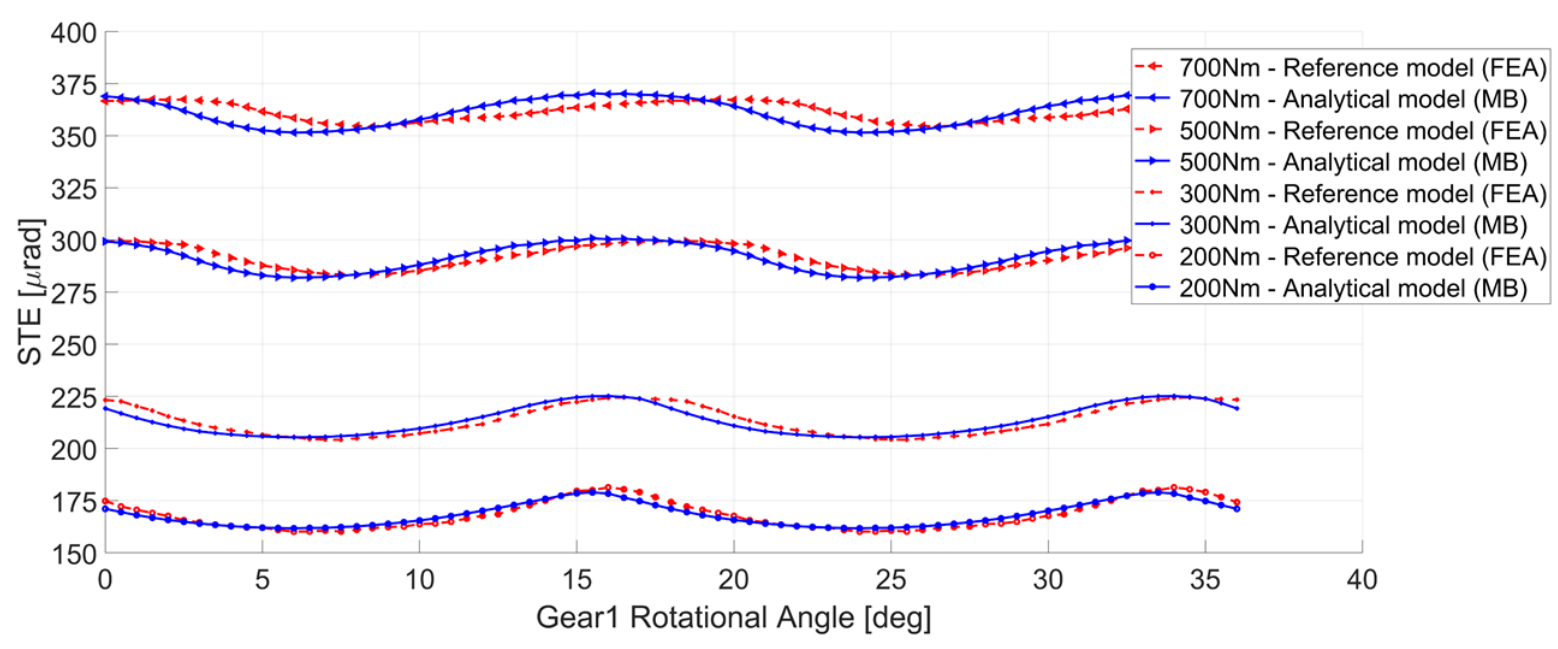

(a) Comparision of the obtained STE curves for moderate to highly loaded conditions $(200 \mathrm{Nm}-300 \mathrm{Nm}-500 \mathrm{Nm}-700 \mathrm{Nm})$.

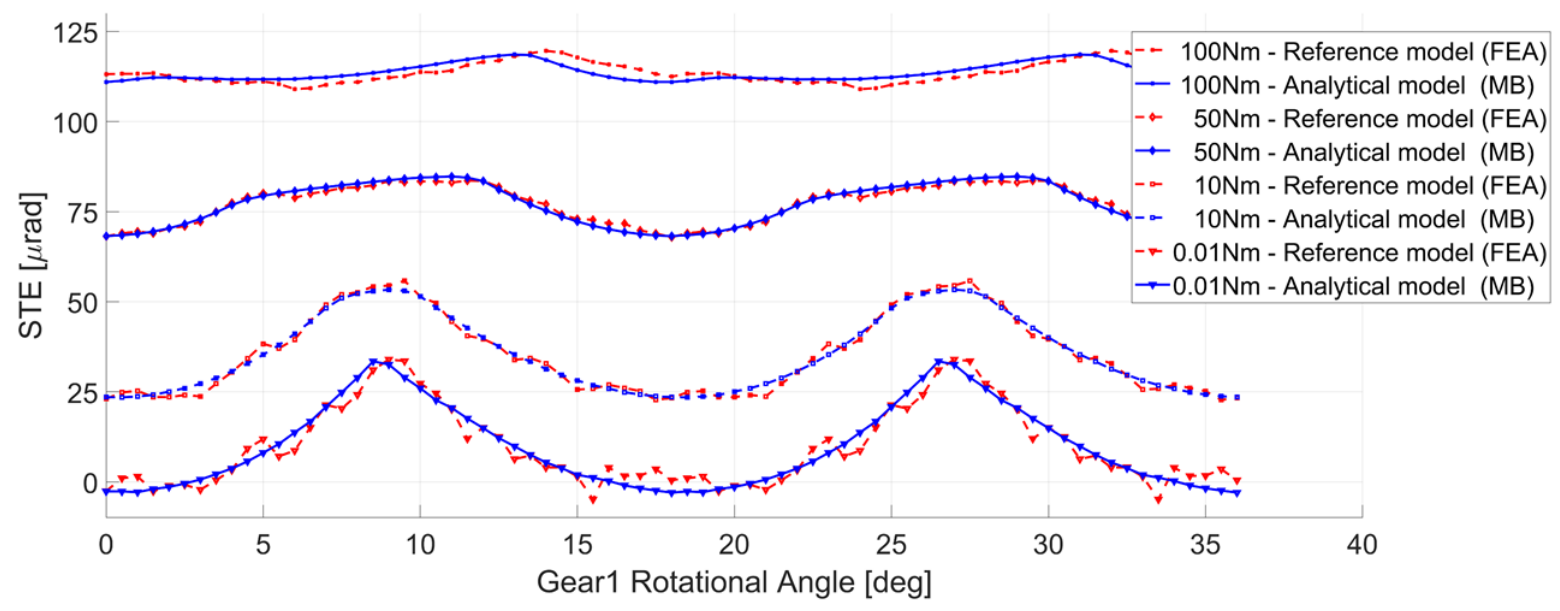

(b) Comparision of the obtained STE curves for unloaded to lowly loaded conditions $(0.01 \mathrm{Nm}-10 \mathrm{Nm}-50 \mathrm{Nm}-100 \mathrm{Nm})$.

Figure 16: Comparison of the static transmission error (STE) curves for reference model (FEA) and analytical model (multibody) for unloaded $(0.01 \mathrm{Nm})$ to highly loaded $(700 \mathrm{Nm})$ conditions.

\section{Conclusions}

The presented work focused on the development and validation of a numerically efficient and accurate methodology for tooth contact analysis of spiral bevel gears, applicable in a multibody framework. The tooth geometry has been defined through simulation of the manufacturing process and was considered to be given prior to the contact simulation in order to develop a generally applicable methodology. The cornerstone of the methodology rests on the fact that the real contacting flanks are designed to transmit motion and are thus nearly conjugate. Using the equation of meshing the roll surface, the action surface and the conjugate to the flank were computed for each real flank of the flank pair.

A multibody approach to tooth contact analysis was then proposed that assumes contact between rigid surfaces. Using the knowledge about the surface of action of each flank, a general, accurate and numerically efficient methodology for contact detection was developed to determine instantaneous curves of potential contact points. Contact between the mating flanks was determined based on the penetration between the rigid flanks and the resulting contact forces are computed under the assumption that the penetration matches with the deformation of the gear teeth, if they were flexible. The tooth compliance has been modeled in an 
analytical fashion and was assumed to be the result of two contributions. The local contribution described the contact deformation due to Hertzian flattening, while the global contribution described the deformation of the tooth outside of the contact zone. Tredgold's approximation in combination with a set expressions, based on beam theory, for spur gear teeth were adapted to approximate the global tooth compliance.

The proposed methodology for contact analysis has been validated against detailed nonlinear finite element contact simulations. The results for peak-to-peak static transmission error, contact pattern and contact pressure distribution that were obtained with the proposed methodology showed good correlation with the nonlinear FE results. Moreover, the computational time of the proposed model showed to be only a fraction of that of the nonlinear FE simulations.

In its current form the proposed model allows for future work to focus on the development of numerically efficient strategies to correctly include gear pair misalignments or to explore more advanced methodologies to better model the global tooth compliance and load sharing. Such contributions would not only allow to further expand its current applicability but also its accuracy. Next to this, the numerically efficiency of the proposed model also allows for further application in optimization studies and even system analysis.

(2009) 1848 - 1865. doi:https://doi.org/10.1016/j.mechmachtheory.2009.03.009.

[11] M. Kolivand, A. Kahraman, An ease-off based method for loaded tooth contact analysis of hypoid gears having local and global surface deviations, Journal of Mechanical Design 132 (2010) 071004-071004-8. doi:10.1115/1.4001722.

[12] Q. Fan, Optimization of face cone element for spiral bevel and hypoid gears, Journal of Mechanical Design 133 (2011) 091002-1-7. doi:10.1115/1.4004546.

[13] A. Artoni, A. Bracci, M. Gabiccini, M. Guiggiani, Optimization of the loaded contact pattern in hypoid gears by automatic topography modification, Journal of Mechanical Design 131 (2009) 011008-1 - 011008-9. doi:10.1115/1.3013844.

[14] A. Artoni, M. Kolivand, A. Kahraman, An ease-off based optimization of the loaded transmission error of hypoid gears, Journal of Mechanical Design 132 (2010) 011010-1 - 011010-9. doi:10.1115/1.4000645.

[15] A. Artoni, M. Gabiccini, M. Guiggiani, A. Kahraman, Multi-objective ease-off optimization of hypoid gears for their efficiency, noise, and durability performances, Journal of Mechanical Design 133 (2011) 121007-1 - 121007-9. doi:10.1115/1.4005234.

[16] A. Artoni, M. Gabiccini, M. Kolivand, Ease-off based compensation of tooth surface deviations for spiral bevel and hypoid gears: Only the pinion needs corrections, Mechanism and Machine Theory 61 (2013) 84 - 101. doi:https://doi.org/10.1016/j.mechmachtheory.2012.10.005. 
[18] E. Wildhaber, Basic relationships of hypoid gears, American Machinist 90 (4) (1946) 108-111.

[19] M. Baxter, Basic geometry and tooth contact of hypoid gears, Ind. Math. 11 (2) (1961) 19-42.

[20] T. J. Krenzer, Understanding tooth contact analysis, The Gleason Works Publication (SD3139B/3-81/GMD).

[21] Klingelnberg, Kimos: Zahnkontakt-analyse für kegelräder, Tech. rep., Klingelnberg Gruppe (1996).

$22]$ M. Kolivand, Development of tooth contact and mechanical efficiency models for face-milled and face-hobbed hypoid and spiral bevel gears., Ph.D. thesis, Graduate School of The Ohio State University (2009).

[23] F. L. Litvin, G. I. Sheveleva, D. Vecchiato, I. Gonzalez-Perez, A. Fuentes, Modified approach for tooth contact analysis of gear drives and automatic determination of guess values, Computer Methods in Applied Mechanics and Engineering 194 (27) (2005) 2927 - 2946. doi:https://doi.org/10.1016/j.cma.2004.07.031

[24] D. He, H. Ding, J. Tang, A new analytical identification approach to the tooth contact points considering misalignments for spiral bevel or hypoid gears, Mechanism and Machine Theory 121 (2018) 785 - 803. doi:https://doi.org/10.1016/j.mechmachtheory.2017.12.003.

[25] H. J. Stadtfeld, Handbook of bevel and hypoid gears: calculation, manufacturing and optimization, Rochester Institute of Technology, 1993.

26] D. B. Dooner, M. Vivet, D. Mundo, Deproximating tredgold's approximation, Mechanism and Machine Theory 102 (2016) 36 - 54. doi:https://doi.org/10.1016/j.mechmachtheory.2016.03.004.

[27] T. J. Krenzer, Tooth contact analysis of spiral bevel and hypoid gears under load, in: SAE Technical Paper, SAE International, 1981. doi:10.4271/810688.

[28] A. H. Elkholy, A. A. Elsharkawy, A. S. Yigit, Effect of meshing tooth stiffness and manufacturing error on the analysis of straight bevel gears., Mechanics of Structures and Machines 26 (1) (1998) 41-61. doi:10.1080/08905459808945419.

[29] T. Nakada, M. Utagawa, The dynamic loads on gears caused by the varying elasticity of the mating teeth, in: Proc. of the 6th Jap. Nat. Cong. for Appl. Mech., 1956.

[30] C. Gosselin, L. Cloutier, Q. Nguyen, A general formulation for the calculation of the load sharing and transmission error under load of spiral bevel and hypoid gears, Mechanism and Machine Theory 30 (3) (1995) 433 - 450. doi:https://doi.org/10.1016/0094-114X(94)00049-Q.

[31] Q. Fan, L. Wilcox, New Developments in Tooth Contact Analysis (TCA) and Loaded TCA for Spiral Bevel and Hypoid Gear Drives, Technical paper / FTM.: Technical paper, AGMA, 2005.

[32] J. E. Hemmelmann, Simulation des lastfreien und belasteten zahneingriffs zur analyse der drehübertragung von zahnradgetrieben, Ph.D. thesis (2007).

3] B. Neupert, Berechnung der zahnkräfte, pressungen und spannungen von stirn- und kegelradgetrieben., Ph.D. thesis (1983).

[34] S. Vaidyanthan, H. Busby, D. Houser, A Rayleigh-Ritz approach to determine compliance and root stresses in spiral bevel gears using shell theory, AGMA, 1993.

[35] R. J. Lisle, J. M. Robinson, The mohr circle for curvature and its application to fold description, Journal of Structural Geology 17 (5) (1995) 739 - 750. doi:https://doi.org/10.1016/0191-8141(94)00089-I.

[36] R. J. Lisle, D. M. Ragan, Strain from three stretches: a simple mohr circle solution, Journal of Structural Geology 10 (8) (1988) 905 - 906. doi:https://doi.org/10.1016/0191-8141(88)90103-4.

[37] M. Vivet, G. Heirman, T. Tamarozzi, W. Desmet, D. Mundo, An ease-off based methodology for contact detection and penetration calculation, in: Power Transmissions: Proceedings of the International Conference on Power Transmissions 2016 (ICPT 2016), , Chongqing University, 2016, pp. 167-173.

[38] Q. Fan, Ease-off and application in tooth contact analysis for face-milled and face-hobbed spiral bevel and hypoid gears, in: Theory and Practice of Gearing and Transmissions, Springer International Publishing, 2015, pp. 321-339. doi:10.1007/9783-319-19740-1_15.

[39] International organization for standardization (ISO), ISO 21771:2007, Gears - Cylindrical Involute Gears and Gear Pairs - Concepts and Geometry, ISO, 2007.

[40] A. A. Shabana, Dynamics of Multibody Systems, 3rd Edition, Cambridge University Press, 2005. doi:10.1017/CBO9780511610523.

[41] M. Géradin, A. Cardona, Flexible multibody dynamics: a finite element approach, John Wiley \& Sons, LTD, 2001.

[42] J. Diebel, Representing attitude: Euler angles, unit quaternions, and rotation vectors (2006).

560 [43] AGMA, 2005-D03 design manual for bevel gears, Tech. rep., American Gear Manufacturers Association (2003).

[44] A. F. del Rincon, F. Viadero, M. Iglesias, P. García, A. de Juan, R. Sancibrian, A model for the study of meshing stiffness in spur gear transmissions, Mechanism and Machine Theory 61 (2013) $30-58$. doi:http://dx.doi.org/10.1016/j.mechmachtheory.2012.10.008.

[45] A. Andersson, L. Vedmar, A dynamic model to determine vibrations in involute helical gears, Journal of Sound and Vibration 260 (2) (2003) 195 - 212. doi:https://doi.org/10.1016/S0022-460X(02)00920-3.

[46] C. Weber, K. Banaschek, Formänderung und profilrücknahme bei gerad-und schrägverzahnten rädern., Tech. rep., VDMA (1955).

[47] K. L. Johnson, Contact mechanics, Cambridge University Press, 1987.

[48] Siemens Product Lifecycle Management Software Inc., NX Nastran 10. advanced nonlinear solution - theory and modeling guide (2014).

[49] P. Wriggers, Computational Contact Mechanics, 2nd Edition, Springer Berlin Heidelberg, 2006. doi:10.1007/978-3-54032609-0. 